\title{
The Query Complexity of Finding a Hidden Permutation
}

\author{
Peyman Afshani ${ }^{1}$, Manindra Agrawal ${ }^{2}$, Benjamin Doerr ${ }^{3}$, \\ Kasper Green Larsen ${ }^{1}$, Kurt Mehlhorn ${ }^{3}$, Carola Winzen ${ }^{3}$ \\ ${ }^{1}$ MADALGO, Department of Computer Science, Aarhus University, Denmark \\ ${ }^{2}$ Indian Institute of Technology Kanpur, India \\ ${ }^{3}$ Max Planck Institute for Informatics, Saarbrücken, Germany
}

\begin{abstract}
We study the query complexity of determining a hidden permutation. More specifically, we study the problem of learning a secret $(z, \pi)$ consisting of a binary string $z$ of length $n$ and a permutation $\pi$ of $[n]$. The secret must be unveiled by asking queries $x \in\{0,1\}^{n}$, and for each query asked, we are returned the score $f_{z, \pi}(x)$ defined as

$$
f_{z, \pi}(x):=\max \left\{i \in[0 . . n] \mid \forall j \leq i: z_{\pi(j)}=x_{\pi(j)}\right\} ;
$$

i.e., the length of the longest common prefix of $x$ and $z$ with respect to $\pi$. The goal is to minimize the number of queries asked.

Our main result are matching upper and lower bounds for this problem, both for deterministic and randomized query schemes. The deterministic query complexity is $\Theta(n \log n)$, which, surprisingly, improves to $\Theta(n \log \log n)$ in the randomized setting.

For the randomized query complexity, both the upper and lower bound are stronger than what can be achieved by standard arguments like the analysis of random queries or information-theoretic considerations. Our proof of the $\Omega(n \log \log n)$ lower bound is based on a potential function argument, which seems to be uncommon in the query complexity literature. We find this potential function technique a very powerful tool in proving lower bounds for randomized query schemes and we expect it to find applications in many other query complexity problems.
\end{abstract}

Keywords: Query complexity; randomized algorithms.

\section{Introduction}

Query complexity, also referred to as decision tree complexity, is one of the most basic models of computation. We aim at learning an unknown object (the secret) by asking queries of a certain type. The cost of the computation is the number of queries made until the secret is unveiled. All other computation is free. Surprisingly, for many simple and natural problems we do not know the number of queries necessary, both if randomization is allowed or not.

In the context of Boolean functions - see [BdW02] for a survey - the goal is to evaluate a given Boolean function $f$ on a secret argument $x$ by querying bits of $x$. For example, $x$ could be the adjacency matrix of a graph and $f$ is one (zero) if the graph is connected (not connected). For graph connectivity the deterministic query complexity is $\Theta\left(n^{2}\right)$ and the randomized complexity is only known to be $\Omega\left(n^{4 / 3} \log ^{1 / 3} n\right)$, cf. [CK07].

It is an open question since Saks' and Wigderson's paper [SW86] from 1986 to determine the randomized query complexity of the recursive 3-Majority function. It asks to determine the value of the root of a complete ternary tree of height $h$. The value of every internal node in the 
tree is the majority value of its three children. We have query access to the $3^{h}$ leaves, which are labeled with binary values. Recent progress include the papers by Jayram, Kumar, and Sivakumar [JKS03] and Magniez, Nayak, Santha, and Xiao [MNSX11]. Magniez et al. provide the current best lower and upper bounds of $(5 / 2)^{h}$ and $(1.004) \cdot 2.64946^{h}$, respectively.

Numerous related - seemingly simple looking problems - of unknown query complexities exist. In the field of property testing, it is open to date how many queries to a Boolean function $f:\{0,1\}^{n} \rightarrow\{0,1\}$ we need to determine with probability $2 / 3$ whether $f$ is a $k$-parity function or far from being one. For non-adaptive testing sequences, Buhrman, García-Soriano, Matsliah, and de Wolf [BGSMdW12] very recently could prove that the query complexity is $\Theta(k \log k)$. For the general, adaptive, case the best known lower bound [BBM12] is $\Omega(k)$, while the best known upper bound is $O(k \log k)$.

Another long-standing open question is the coin weighing problem. Here we are given $n$ coins of two different weights and a spring scale. Our goal is to classify the coins into light and heavy ones. We may use the spring scale to weigh arbitrary subsets of the coins. What is the smallest number of weighings needed, in the worst-case, to classify the coins? This problem remains open since 1963 when Erdős and Rényi [ER63] showed a a lower bound of $(1+o(1)) n / \log _{2} n$ and an upper bound of $(1+o(1))\left(\log _{2} 9\right) n / \log _{2} n$. The upper bound was subsequently improved to $(1+o(1)) 2 n / \log _{2} n$ by Lindström [Lin65] and, independently, by Cantor and Mills [CM66], but no tight bound is known to date. A number of different versions of coin weighing problems exist. A good survey can be found in Bshouty's paper on polynomial time query-optimal algorithms for coin weighing problems [Bsh09].

A related open problem is the query complexity of the Mastermind game with $n$ positions and $k=n$ colors. In Mastermind, the secrets and queries are strings of length $n$ over a $k$-ary alphabet. If the secret is $z$, a query $x$ returns the number eq $(z, x):=\left|\left\{i \mid x_{i}=z_{i}\right\}\right|$ of positions in which $x$ and the secret code $z$ coincide. Although the asymptotically best upper bound of Chvátal [Chv83] was recently improved from $O(n \log n)$ to $O(n \log \log n)$, cf. [DSTW13], the best known lower bound is the trivial linear one. This problem is open for more than 30 years. For both the coin weighing problem of Erdős and Rényi as well as for the Mastermind problem of Chvátal it is widely believed that the current lower bounds are not optimal.

Our Contribution In this work, we shift the focus from searching for secret bits (or bitstrings) to hidden permutations. Apart from the classic result that $\Theta(n \log n)$ comparison queries are necessary and sufficient for sorting, we are not aware of any other query complexity theoretic work on finding permutations.

The query problem we regard originates from theoretical work on randomized search heuristics (see the end of this section), but seems to be a good first example for studying permutation query problems. In particular, it nicely captures the aspect of order/sequentiality in that $\pi(i)$ is easier to detect than $\pi(j)$ when $i<j$.

Let $S_{n}$ denote the set of permutations of $[n]:=\{1, \ldots, n\}$; let $[0 . . n]:=\{0,1, \ldots, n\}$. Our problem is that of learning a hidden permutation $\pi \in S_{n}$ together with a hidden bit-string $z \in\{0,1\}$ through (as few as possible) queries of the following type. A query is again a bitstring $x \in\{0,1\}^{n}$, as answer we receive the length of the longest common prefix of $x$ and $z$ in the order of $\pi$, which we denote by

$$
f_{z, \pi}(x):=\max \left\{i \in[0 . . n] \mid \forall j \leq i: z_{\pi(j)}=x_{\pi(j)}\right\} .
$$

We call this problem the HidDENPERMUTATION problem.

We show the following.

(1) We exhibit a randomized strategy that finds the secret with an expected number of $O(n \log \log n)$ queries (Section 4). This improves upon the $O(n \log n / \log \log n)$ bound 


\section{from [DW12].}

The $O(n \log \log n)$ bound is quite remarkable. Putting it into an information-theoretic perspective, this result shows that we succeed in learning an average of $\Omega(\log n / \log \log n)$ bits of information in each query. This in particular means that our queries allow many different answers to show up with sufficiently high probability. Hence we partially overcome the slowness of learning one $\left(z_{\pi(i)}, \pi(i)\right)$ after the other in the order $i=1, \ldots, n$.

Our strategy is efficient; i.e., it can be implemented in polynomial time.

(2) We then show that this upper bound is asymptotically optimal (Section 5). The lower bound is derived from a potential function argument, which, as far as we know, seems uncommon in the query complexity literature. We believe this potential function approach is very interesting in its own right, and we expect that it may be applied to many other query complexity problems to yield improved lower bounds.

The lower bound is particularly interesting as for many related problems (sorting, Mastermind, and many coin weighing problems, the asymptotic query complexity or the best known lower bound equals the information-theoretic lower bound. This is not the case here, where the information-theoretic lower bound is linear (cf. Section 2 for a sketch). This also was the best lower bound known so far.

(3) Interestingly, and unlike for the problems mentioned in the previous paragraph, the deterministic query complexity of our problem is asymptotically larger than the randomized one. In Section 3, we prove a lower bound of $\Omega(n \log n)$ queries for any deterministic query scheme, which is easily proven tight by sequentially determining $\left(z_{\pi(i)}, \pi(i)\right)$ for $i=1, \ldots, n$.

Origin of the Problem The problem we regard in this work has its origins in the field of evolutionary algorithms. Here, the so-called LEADINGOnEs function

$$
\{0,1\}^{n} \rightarrow[0 . . n], x \mapsto \max \left\{i \in[0 . . n] \mid \forall j \leq i: x_{j}=1\right\}
$$

is a commonly used testbed both for experimental and theoretical analyses (e.g., [Rud97]). It turns out that many genetic and evolutionary algorithms as well as other general-purpose randomized search heuristics used in practice query the fitness of $\Theta\left(n^{2}\right)$ solution candidates until they find the maximum of LEADINGOnES, see, e.g., [DJW02]. Naturally, the same is true when LEADINGONES is replaced by any function LEADINGONES $\circ \sigma$, where $\sigma$ is an automorphism of the $n$-dimensional cube.

In an attempt to understand the intrinsic difficulty of a problem for general purpose search heuristics, Droste, Jansen and Wegener [DJW06] suggested a query complexity theoretic notion called black-box complexity, which roughly speaking (see [DJW06] for the details) is the number of search points a black-box optimization algorithm has to evaluate to find the optimum of an unknown optimization instance from a known problem. The connection to our work is made by the easy observation that the black-box complexity of the class of all LEADINGONES $\circ \sigma$ functions is asymptotically equal to the randomized query complexity of our HIDDENPERMUTATION problem. For this reason, the black-box complexity result in [DW12] implies an upper bound of $O(n \log n / \log \log n)$ for the randomized query complexity of our problem.

\section{Preliminaries}

For all positive integers $k \in \mathbb{N}$ we define $[k]:=\{1, \ldots, k\}$ and $[0 . . k]:=[k] \cup\{0\}$. By $e_{k}^{n}$ we denote the $k$ th unit vector $(0, \ldots, 0,1,0, \ldots, 0)$ of length $n$. For a set $I \subseteq[n]$ we define $e_{I}^{n}:=\sum_{i \in I} e_{i}^{n}=\oplus_{i \in I} e_{i}^{n}$, where $\oplus$ denotes the bitwise exclusive-or. We say that we create $y$ from $x$ by flipping $I$ or that we create $y$ from $x$ by flipping the entries in position(s) $I$ if $y=x \oplus e_{I}^{n}$. 
By $S_{n}$ we denote the set of all permutations of $[n]$. For $r \in \mathbb{R}_{\geq 0}$, let $\lceil r\rceil:=\min \left\{n \in \mathbb{N}_{0} \mid n \geq r\right\}$. and $\lfloor r\rfloor:=\max \left\{n \in \mathbb{N}_{0} \mid n \leq r\right\}$. To increase readability, we sometimes omit the $\lceil\cdot\rceil$ signs; that is, whenever we write $r$ where an integer is required, we implicitly mean $\lceil r\rceil$.

Let $n \in \mathbb{N}$. For $z \in\{0,1\}^{n}$ and $\pi \in S_{n}$ we define

$$
f_{z, \pi}:\{0,1\}^{n} \rightarrow[0 . . n], x \mapsto \max \left\{i \in[0 . . n] \mid \forall j \leq i: z_{\pi(j)}=x_{\pi(j)}\right\} .
$$

$z$ is called the target string of $f_{z, \pi}$ and $\pi$ is called the target permutation. In the HidDENPERMUTATION problem we want to identify target string and target permutation by asking queries $x^{i}$ and evaluating the answers ("scores") $s^{i}=f_{z, \pi}\left(x^{i}\right)$. We may stop after $t$ queries if there is only a single pair $(z, \pi) \in\{0,1\}^{n} \times S_{n}$ with $s^{i}=f_{z, \pi}\left(x^{i}\right)$ for $1 \leq i \leq t$.

A randomized strategy for the HIDDENPERMUTATION problem is a tree of outdegree $n+1$ in which a probability distribution over $\{0,1\}^{n}$ is associated with every node of the tree. The search starts as the root. In any node, the query is selected according to the probability distribution associated with the node, and the search proceeds to the child selected by the score. The complexity of a strategy on input $(z, \pi)$ is the expected number of queries required to identify the secret, and the randomized query complexity of a strategy is the worst case over all secrets. Deterministic strategies are the special case in which the probability distributions are one-point distributions; i.e., a fixed query is associated with every node. The deterministic (randomized) query complexity of HidDENPERMUTATION is the best possible (expected) complexity.

We remark that knowing $z$ allows us to determine $\pi$ with $n-1$ queries $z \oplus e_{i}^{n}, 1 \leq i<n$. Observe that $\pi^{-1}(i)$ equals $f_{z, \pi}\left(z \oplus e_{i}^{n}\right)+1$. Conversely, knowing the target permutation $\pi$ we can identify $z$ in a linear number of guesses. If our query $x$ has a score of $k$, all we need to do next is to query the string $x^{\prime}$ that is created from $x$ by flipping the entry in position $\pi(k+1)$. Thus, learning one part of the secret is no easier (up to $O(n)$ questions) than learning the full.

A simple information-theoretic argument gives an $\Omega(n)$ lower bound for the deterministic query complexity and, together with Yao's minimax principle [Yao77], also for the randomized complexity. The search space has size $2^{n} n$ !, since the unknown secret is an element of $\{0,1\}^{n} \times$ $S_{n}$. That is, we need to "learn" $\Omega(n \log n)$ bits of information. Each score is a number between 0 and $n$, i.e., we learn at most $O(\log n)$ bits of information per query, and the $\Omega(n)$ bound follows.

Let $\mathcal{H}:=\left(x^{i}, s^{i}\right)_{i=1}^{t}$ be a vector of queries $x^{i} \in\{0,1\}^{n}$ and scores $s^{i} \in[0 . . n]$. We call $\mathcal{H}$ a guessing history. A secret $(z, \pi)$ is consistent with $\mathcal{H}$ if $f_{z, \pi}\left(x^{i}\right)=s^{i}$ for all $i \in[t]$. $\mathcal{H}$ is feasible if there exists a secret consistent with it.

An observation crucial in our proofs is the fact that a vector $\left(V_{1}, \ldots, V_{n}\right)$ of subsets of $[n]$, together with a top score query $\left(x^{*}, s^{*}\right)$, captures the total knowledge provided by a guessing history $\mathcal{H}=\left(x^{i}, s^{i}\right)_{i=1}^{t}$ about the set of secrets consistent with $\mathcal{H}$. We will call $V_{j}$ the candidate set for position $j ; V_{j}$ will contain all indices $i \in[n]$ for which the following simple rules (1) to (3) do not rule out that $\pi(j)$ equals $i$.

Theorem 1. Let $t \in \mathbb{N}$, and let $\mathcal{H}=\left(x^{i}, s^{i}\right)_{i=1}^{t}$ be a guessing history. Construct the candidate sets $V_{1}, \ldots, V_{n} \subseteq[n]$ according to the following rules:

(1) If there are $h$ and $\ell$ with $j \leq s^{h} \leq s^{\ell}$ and $x_{i}^{h} \neq x_{i}^{\ell}$, then $i \notin V_{j}$.

(2) If there are $h$ and $\ell$ with $s=s^{h}=s^{\ell}$ and $x_{i}^{h} \neq x_{i}^{\ell}$, then $i \notin V_{s+1}$.

(3) If there are $h$ and $\ell$ with $s^{h}<s^{\ell}$ and $x_{i}^{h}=x_{i}^{\ell}$, then $i \notin V_{s^{h}+1}$.

(4) If $i$ is not excluded by one of the rules above, then $i \in V_{j}$.

Furthermore, let $s^{*}:=\max \left\{s^{1}, \ldots, s^{t}\right\}$ and let $x^{*}=x^{j}$ for some $j$ with $s^{j}=s^{*}$.

Then a pair $(z, \pi)$ is consistent with $\mathcal{H}$ if and only if (a) $f_{z, \pi}\left(x^{*}\right)=s^{*}$ and $(b) \pi(j) \in V_{j}$ for all $j \in[n]$. 
Proof. Let $(z, \pi)$ satisfy conditions (a) and (b). We show that $(z, \pi)$ is consistent with $\mathcal{H}$. To this end, let $h \in[t]$, let $x=x^{h}, s=s^{h}$, and $f:=f_{z, \pi}(x)$. We need to show $f=s$.

Assume $f<s$. Then $z_{\pi(f+1)} \neq x_{\pi(f+1)}$. Since $f+1 \leq s^{*}$, this together with (a) implies $x_{\pi(f+1)} \neq x_{\pi(f+1)}^{*}$. Rule (1) yields $\pi(f+1) \notin V_{f+1}$; a contradiction to (b).

Similarly, if we assume $f>s$, then $x_{\pi(s+1)}=z_{\pi(s+1)}$. We distinguish two cases. If $s<s^{*}$, then by condition (a) we have $x_{\pi(s+1)}=x_{\pi(s+1)}^{*}$. By rule (3) this implies $\pi(s+1) \notin V_{s+1}$; a contradiction to (b).

On the other hand, if $s=s^{*}$, then $x_{\pi(s+1)}=z_{\pi(s+1)} \neq x_{\pi(s+1)}^{*}$ by (a). Rule (2) implies $\pi(s+1) \notin V_{\pi(s+1)}$, again contradicting (b).

Necessity is trivial.

We may also construct the sets $V_{j}$ incrementally. The following update rules are direct consequences of Theorem 1. In the beginning, let $V_{j}:=[n], 1 \leq j \leq n$. After the first query, record the first query as $x^{*}$ and its score as $s^{*}$. For all subsequent queries, do the following: Let $I$ be the set of indices in which the current query $x$ and the current best query $x^{*}$ agree. Let $s$ be the objective value of $x$ and let $s^{*}$ be the objective value of $x^{*}$.

Rule 1: If $s<s^{*}$, then $V_{i} \leftarrow V_{i} \cap I$ for $1 \leq i \leq s$ and $V_{s+1} \leftarrow V_{s+1} \backslash I$.

Rule 2: If $s=s^{*}$, then $V_{i} \leftarrow V_{i} \cap I$ for $1 \leq i \leq s^{*}+1$.

Rule 3: If $s>s^{*}$, then $V_{i} \leftarrow V_{i} \cap I$ for $1 \leq i \leq s^{*}$ and $V_{s^{*}+1} \leftarrow V_{s^{*}+1} \backslash I$. We further replace $s^{*} \leftarrow s$ and $x^{*} \leftarrow x$.

It is immediate from the update rules that the $V_{j}$ s form a laminar family; i.e., for $i<j$ either $V_{i} \cap V_{j}=\emptyset$ or $V_{i} \subseteq V_{j}$. As a consequence of Theorem 1 we obtain a polynomial time test for the feasibility of histories. It gives additional insight in the meaning of the candidate sets $V_{1}, \ldots, V_{n}$.

Theorem 2. It is decidable in polynomial time whether a guessing history is feasible. Furthermore, we can efficiently compute the number of pairs consistent with it.

(sketch. A full proof is in the appendix). Let $V_{1}, \ldots, V_{n}$ be as in Theorem 1 . We construct a bipartite graph $G=G\left(V_{1}, \ldots, V_{n}\right)$ with node set $[n]$ on both sides. Connect $j$ to all elements in $V_{j}$ on the other side. Permutations $\pi$ with $\pi(j) \in V_{j}$ for all $j$ are in one-to-one correspondence to perfect matchings in $G$. If there is no perfect matching, the history is infeasible. Otherwise, let $\pi$ be any permutation with $\pi(j) \in V_{j}$ for all $j$. Set $z_{\pi(i)}:=x_{\pi(i)}^{*}$ for $i \in\left[s^{*}\right]$, and $z_{\pi(i)}:=1-x_{\pi(i)}^{*}$ for $i \notin\left[s^{*}\right]$. The pair $(z, \pi)$ is consistent with $\mathcal{H}$.

For the counting problem we first observe that for any fixed consistent permutation $\pi$, the number of strings $z$ such that $(z, \pi)$ is consistent with $\mathcal{H}$ equals $2^{n-\left(s^{*}+1\right)}$ if $s^{*}<n$, and it equals one if $s^{*}=n$. By Hall's condition a perfect matching exists if and only if $\left|\bigcup_{j \in J} V_{j}\right| \geq|J|$ for every $J \subseteq[n]$. Constructing the permutations in a greedy fashion, one sees that the number of consistent permutations is equal to $\prod_{1 \leq i \leq n}\left(\left|V_{i}\right|-\left|\left\{j<i \mid V_{j} \subseteq V_{i}\right\}\right|\right)$.

The fact that the $V_{j}$ s are a laminar family is crucial for the counting result. Counting the number of perfect matching in a general bipartite graph is \#P-complete.

\section{Deterministic Complexity}

In this section, we present simple proofs that completely settle the deterministic query complexity of the HiddenPermutation problem. Specifically, we prove

Theorem 3. The deterministic query complexity of the HiddenPermutation problem with $n$ positions is $\Theta(n \log n)$. 
The lower bound is proved by examining the decision tree of the deterministic query scheme and finding an input for which the number of queries asked is high. More precisely, we show that for every deterministic strategy, there exists an input $(z, \pi)$ such that after $\Omega(n \log n)$ queries the maximal score ever returned is at most $n / 2$. This is done as follows: First consider the root node $r$ of the decision tree. There is one child for every possible score $0,1, \ldots, n$. The crucial observation in the following steps is that there is always one of the two children corresponding to scores 0 and 1 for which the size of the candidate set $V_{1}$ at most halves and $V_{3}, \ldots, V_{n}$ do not change when following the update rules from the preceding section. That is, for any query asked in the first round, there is an answer (either 0 or 1 ) to it such that the number of possible values of $\pi(1)$ at most halves. Thus we recurse into that child on the subset of inputs consistent with that answer. We may continue this for $\Omega(\log n)$ steps, until $\left|V_{1}\right|=2$. At this point, let $v$ be the node reached in the decision tree and let $x^{*}$ be an arbitrary one of the queries asked which achieved the maximal score (remember the maximal score is either 0 or 1 ). Now choose $i^{\prime} \in V_{1}$ and $i^{\prime \prime} \in V_{2}$ arbitrarily and consider the subset of all inputs for which $i^{\prime}=\pi(1), i^{\prime \prime}=\pi(2)$, $z_{i^{\prime}}=x_{i^{\prime}}^{*}$, and $z_{i^{\prime \prime}}=1-x_{i^{\prime \prime}}^{*}$. For all such inputs, the query path followed in the decision tree must start by descending from the root to the node $v$. For this collection of inputs, observe that there is one input for every assignment of values to $\pi(3), \ldots, \pi(n)$ different from $i^{\prime}$ and $i^{\prime \prime}$, and for every assignment of $0 / 1$ values to $z_{\pi(3)}, \ldots, z_{\pi(n)}$. Hence we can recurse on this subset of inputs starting at $v$ ignoring $V_{1}, V_{2}, \pi(1), \pi(2), z_{\pi(1)}$, and $z_{\pi(2)}$. The setup is identical to what we started out with at the root, with the problem size decreased by 2 . We proceed this way, forcing $\Omega(\log n)$ queries for every two positions revealed, until we have returned a score of $n / 2$ for the first time. At this point, we have forced at least $n / 4 \cdot \Omega(\log n)=\Omega(n \log n)$ queries.

The upper bound is achieved by an algorithm that resembles binary search and iteratively identifies $\pi(1), \ldots, \pi(n)$ and the corresponding bit values $z_{\pi(1)}, \ldots, z_{\pi(n)}$ : We start by querying the all-zeros string $(0, \ldots, 0)$ and the all-ones string $(1, \ldots, 1)$. The scores determine $z_{\pi(1)}$. By flipping a set $I$ containing half of the bit positions in the better (the one achieving largest score) of the two strings, we can determine whether $\pi(1) \in I$ or not. This allows us to find $\pi(1)$ via a binary search strategy in $O(\log n)$ queries. Once $\pi(1)$ and $z_{\pi(1)}$ are known, we iterate this strategy on the remaining bit positions to determine $\pi(2)$ and $z_{\pi(2)}$, and so on, yielding an $O(n \log n)$ query strategy for identifying the secret, cf. Section B.2.

\section{The Randomized Strategy}

We now show that the randomized query complexity is only $O(n \log \log n)$. This implies that we found a way to overcome the sequential learning process of the binary search strategy (typically revealing a constant amount of information per query) and instead have a typical information gain of $\Theta(\log n / \log \log n)$ bits per query. In the language of the candidate sets $V_{i}$, we manage to reduce the sizes of many $V_{i}$ s in parallel, that is, we gain information on several $\pi(i)$ s despite the seemingly sequential way $f_{z, \pi}$ offers information. The key to this is using partial information given by the $V_{i}$ (that is, information that does not determine $\pi_{i}$, but only restricts it) to guess with good probability an $x$ with $f_{z, \pi}(x)>s^{*}$.

Theorem 4. The randomized query complexity of the HidDENPERMUTATION problem with $n$ positions is $O(n \log \log n)$.

The strategy has two parts. In the main part, we identify the positions $\pi(1), \ldots, \pi(q)$ and the corresponding bit values $z_{\pi(1)}, \ldots, z_{\pi(q)}$ for some $q \in n-\Theta(n / \log n)$ with $O(n \log \log n)$ queries. In the second part, we find the remaining $n-q \in \Theta(n / \log n)$ positions and entries using the binary search algorithm with $O(\log n)$ queries per position. Part 1 is outlined below; the details are in the appendix. 


\subsection{The Main Strategy}

Here and in the following we denote by $s^{*}$ the current best score, and by $x^{*}$ we denote a corresponding query; i.e., $f_{z, \pi}\left(x^{*}\right)=s^{*}$. For brevity, we write $f$ for $f_{z, \pi}$.

There is a trade-off between learning more information about $\pi$ by reducing the sets $V_{1}, \ldots, V_{s^{*}+1}$ and increasing the score $s^{*}$. Our main task is to find the right balance between the two. In our $O(n \log \log n)$ strategy, we partition the sets $V_{1}, \ldots, V_{n}$ into several levels, each of which has a certain capacity. Depending on the status (i.e., the fill rate) of these levels, either we try to increase $s^{*}$, or we aim at reducing the sizes of the candidate sets.

In the beginning, all candidate sets $V_{1}, \ldots, V_{n}$ belong to level 0 . In the first step we aim at moving $V_{1}, \ldots, V_{\log n}$ to the first level. This is done sequentially. We start by querying $f(x)$ and $f(y)$, where $x$ is arbitrary and $y=x \oplus 1^{n}$ is the bitwise complement of $x$. By swapping $x$ and $y$ if needed, we may assume $f(x)=0<f(y)$. We now run a randomized binary search for finding $\pi(1)$. We choose uniformly at random a subset $F_{1} \subseteq V_{1}\left(V_{1}=[n]\right.$ in the beginning) of size $\left|F_{1}\right|=\left|V_{1}\right| / 2$. We query $f\left(y^{\prime}\right)$ where $y^{\prime}$ is obtained from $y$ by flipping the bits in $F_{1}$. If $f\left(y^{\prime}\right)>f(x)$, we set $V_{1} \leftarrow V_{1} \backslash F_{1}$; we set $V_{1} \leftarrow F_{1}$ otherwise. This ensures $\pi(1) \in V_{1}$. We stop this binary search once $\pi(2) \notin V_{1}$ is sufficiently likely; the analysis will show that $\operatorname{Pr}\left[\pi(2) \in V_{1}\right] \leq 1 / \log ^{d} n$ (and hence $\left|V_{1}\right| \leq n / \log ^{d} n$ ) for some large enough constant $d$ is a good choice.

We now start pounding on $V_{2}$. Let $\{x, y\}=\left\{y, y \oplus 1_{[n] \backslash V_{1}}\right\}$. If $\pi(2) \notin V_{1}$, one of $f(x)$ and $f(y)$ is one and the other is larger than one. Swapping $x$ and $y$ if necessary, we may assume $f(x)=1<f(y)$. We now use randomized binary search to reduce the size of $V_{2}$ to $n / \log ^{d} n$. The randomized binary search is similar to before. Initially we have $V_{2}=[n] \backslash V_{1}$. At each step we chose a subset $F_{2} \subseteq V_{2}$ of size $\left|V_{2}\right| / 2$ and we create $y^{\prime}$ from $y$ by flipping the bits in positions $F_{2}$. If $f\left(y^{\prime}\right)=1$ we update $V_{2}$ by $F_{2}$ and we update $V_{2}$ by $V_{2} \backslash F_{2}$ otherwise. We stop once $\left|V_{2}\right| \leq n / \log ^{d} n$.

At this point we have $\left|V_{1}\right|,\left|V_{2}\right| \leq n / \log ^{d} n$ and $V_{1} \cap V_{2}=\emptyset$. We hope that $\pi(3) \notin V_{1} \cup V_{2}$, in which case we move set $V_{3}$ from level 0 to level 1 (we comment on the case $\pi(3) \in V_{1} \cup V_{2}$ in the failure section, Section 4.2).

At some point the probability that $\pi(i) \notin V_{1} \cup \ldots \cup V_{i-1}$ drops below a certain threshold and we cannot ensure to make progress anymore by simply querying $y \oplus\left([n] \backslash\left(V_{1} \cup \ldots \cup V_{i-1}\right)\right)$. This situation is reached when $i=\log n$ and hence we abandon the previously described strategy once $s^{*}=\log n$. At this point, we move our focus from increasing the current best score $s^{*}$ to reducing the size of the candidate sets $V_{1}, \ldots, V_{s^{*}}$, thus adding them to the second level. More precisely, we reduce their sizes to at most $n / \log ^{2 d} n$. This reduction is carried out by subroutine2, which we describe in Section 4.3.

Once the sizes $\left|V_{1}\right|, \ldots,\left|V_{s^{*}}\right|$ have been reduced to at most $n / \log ^{2 d} n$, we move our focus back to increasing $s^{*}$. The probability that $\pi\left(s^{*}+1\right) \in V_{1} \cup \ldots \cup V_{s^{*}}$ will now be small enough (details below), and we proceed as before by flipping $[n] \backslash\left(V_{1} \cup \ldots \cup V_{s^{*}}\right)$ and reducing the size of $V_{s^{*}+1}$ to $n / \log ^{d} n$. Again we iterate this process until the first level is filled; i.e., until we have $s^{*}=2 \log n$. As we did with $V_{1}, \ldots, V_{\log n}$, we reduce the sizes of $V_{\log n+1}, \ldots, V_{2} \log n$ to $n / \log ^{2 d} n$, thus adding them to the second level. We iterate this process of moving $\log n$ sets from level 0 to level 1 and then moving them to the second level until $\log ^{2} n$ sets have been added to the second level. At this point the second level has reached its capacity and we proceed by reducing the sizes of $V_{1}, \ldots, V_{\log ^{2} n}$ to at most $n / \log ^{4 d} n$, thus adding them to the third level.

In total we have $t=O(\log \log n)$ levels. For $1 \leq i \leq t$, the $i$ th level has a capacity of $x_{i}:=\log ^{2^{i-1}} n$ sets, each of which is required to be of size at most $n / x_{i}^{d}$. Once level $i$ has reached its capacity, we reduce the size of the sets on the $i$ th level to at most $n / x_{i+1}^{d}$, thus moving them from level $i$ to level $i+1$. When $x_{t}$ sets $V_{i}, \ldots, V_{i+x_{t}}$ have been added to the last 
level, level $t$, we finally reduce their sizes one. This corresponds to determining $\pi(i+j)$ for each $j \in\left[x_{t}\right]$.

This concludes the presentation of the main ideas of the first phase. We still need to discuss how to handle failures, how to move sets from some level $i$ to level $i+1$ (subroutine2), and we need to compute the query complexity of our algorithm.

\subsection{Failures}

We say that a failure happens if we want to move some set $V_{j+1}$ from level 0 to level 1 , but either $f(y)=f\left(y \oplus 1_{[n] \backslash\left\{V_{1} \cup \ldots \cup V_{j}\right\}}\right)=j$ or $f(y), f\left(y \oplus 1_{[n] \backslash\left\{V_{1} \cup \ldots \cup V_{j}\right\}}\right)>j$ holds. In this case we can conclude that $\pi(j+1) \in V_{1} \cup \ldots \cup V_{j}$. Therefore, we immediately abort the first level. That is, we move all sets on the first level to the second one. As before, this is done by calls to subroutine 2 which reduce the size of the sets from at most $n / \log ^{d} n$ to at most $n / \log ^{2 d} n$. We test whether we now have $\left\{f(y), f\left(y \oplus 1_{[n] \backslash\left\{V_{1} \cup \ldots \cup V_{j}\right\}}\right\}=\{j, j+\ell\}\right.$ for some $\ell \geq 1$. Should we still have $\pi(j+1) \in V_{1} \cup \ldots \cup V_{j}$, we continue by moving all level 2 sets to level 3 , and so on, until we finally have $\pi(j+1) \notin V_{1} \cup \ldots \cup V_{j}$. At this point, we proceed again by moving sets from level 0 to level 1 , starting of course with set $V_{j+1}$.

The pseudo-code summarizing phase 1 can be found in the appendix, cf. Algorithm 1.

\subsection{Subroutine2}

We now describe how to reduce the sizes of the up to $x_{\ell-1}$ candidate sets from some value $\leq n / x_{\ell-1}^{d}$ to the target size $n / x_{\ell}^{d}$ of level $\ell$ with an expected number of $O(1) x_{\ell-1} d\left(\log \left(x_{\ell}\right)-\right.$ $\left.\log \left(x_{\ell-1}\right)\right) / \log \left(x_{\ell-1}\right)$ queries. For this reduction we introduce procedure subroutine2: It reduces the sizes of at most $k$ candidate sets to a $k$ th fraction of their original size using at most $O(k)$ queries ( $k$ is a parameter of the routine). We use subroutine2 with parameter $k=x_{\ell-1}$ repeatedly to achieve the full reduction of the sizes to at most $n / x_{\ell}^{d}$.

subroutine 2 is given a set $J$ of at most $k$ indices and a string $y$ with $f(y) \geq \max J$. The goal is to reduce the size of each candidate set $V_{j}, j \in J$, below a target size $m$ where $m \geq\left|V_{j}\right| / k$ for all $j \in J$. The routine works in phases of several iterations each. Let $J$ be the set of indices of the candidate sets that are still above the target size at the beginning of an iteration. For each $j \in J$, we randomly choose a subset $F_{j} \subseteq V_{j}$ of size $\left|V_{j}\right| / k$. We create a new bit string $y^{\prime}$ from $y$ by flipping the entries in positions $\cup_{j \in J} F_{j}$. A condition on the sets $V_{j}, j \in J$, (see Lemma 5) ensures that we have either $f\left(y^{\prime}\right) \geq \max J$ or $f\left(y^{\prime}\right)=j-1$ for some $j \in J$. In the first case, i.e., if $f\left(y^{\prime}\right) \geq \max J$, none of the sets $V_{j}$ was hit, and for all $j \in J$ we can remove the subset $F_{j}$ from the candidate set $V_{j}$. We call such queries "off-trials". An off-trial reduces the size of all sets $V_{j}, j \in J$, to a $(1-1 / k)$ th fraction of their original size. If, on the other hand, we have $f\left(y^{\prime}\right)=j-1$ for some $j \in J$, we can replace $V_{j}$ by set $F_{j}$ as $\pi(j) \in F_{j}$ must hold. Since $\left|F_{j}\right|=\left|V_{j}\right| / k \leq m$ by assumption, this set has now been reduced to its target size and we can remove it from $J$.

We continue in this way until at least half of the indices are removed from $J$ and at least $c k$ off-trials occurred, for some constant $c$ satisfying $(1-1 / k)^{c k} \leq 1 / 2$. We then proceed to the next phase. Consider any $j$ that is still in $J$. The size of $V_{j}$ was reduced by a factor $(1-1 / k)$ at least $c k$ times. Thus its size was reduced to at most half its original size. We may thus halve $k$ without destroying the invariant $m \geq\left|V_{j}\right| / k$ for $j \in J$. The effect of halving $k$ is that the relative size of the sets $F_{j}$ will be doubled for the sets $V_{j}$ that still take part in the reduction process.

The pseudo-code of subroutine2 can be found in the appendix, cf. Algorithm 4 in Section B.3. 
Lemma 5. Let $k \in \mathbb{N}$ and let $J \subseteq[n]$ be a set of at most $k$ indices with $V_{j} \cap V_{p}=\emptyset$ for $j \in J$ and $p \in[\max J] \backslash\{j\}$. Let $y \in\{0,1\}^{n}$ be such that $f(y) \geq \max J$ and let $m \in \mathbb{N}$ be such that $m \geq\left|V_{j}\right| / k$ for all $j \in J$.

In expectation it takes $O(k)$ queries until subroutine2 $(k, J, m, y)$ has reduced the size of $V_{j}$ to at most $m$ for each $j \in J$.

Using successive calls to subroutine2 we get the following corollary.

Corollary 6. Let $k \in \mathbb{N}, J$, and $y$ be as in Lemma 4. Let further $d \in \mathbb{N}$ and $x \in \mathbb{R}$ such that $\max _{j \in J}\left|V_{j}\right|=n / x^{d}$. Let $y \in \mathbb{R}$ with $y>x$.

Using at most $d(\log y-\log x) / \log k$ calls to subroutine2 we can reduce the maximal size $\max _{j \in J}\left|V_{j}\right|$ to $n / y^{d}$. The overall expected number of queries needed to achieve this reduction is $O(1) k d(\log y-\log x) / \log k$.

\subsection{Proof of Theorem 4}

It remains to show that the first phase of our strategy requires at most $O(n \log \log n)$ queries. If no failure happened, the expected number of queries was bounded by

$$
\begin{aligned}
& \frac{q}{x_{t}}\left(\frac{x_{t} \log n}{\log x_{t}}+\frac{x_{t}}{x_{t-1}}\left(\frac{x_{t-1} d c\left(\log x_{t}-\log x_{t-1}\right)}{\log x_{t-1}}\right.\right. \\
& \left.\left.+\frac{x_{t-1}}{x_{t-2}}\left(\ldots+\frac{x_{2}}{x_{1}}\left(\frac{x_{1} d c\left(\log x_{2}-\log x_{1}\right)}{\log x_{1}}+x_{1} d \log x_{1}\right)\right)\right)\right) \\
\leq & n d c\left(\frac{\log n}{\log x_{t}}+\frac{\log x_{t}}{\log x_{t-1}}+\ldots+\frac{\log x_{2}}{\log x_{1}}+\log x_{1}-t\right),
\end{aligned}
$$

where $c$ is the constant hidden in the $O(1)$-term in Lemma 5 . To verify this formula, observe that we fill the $(i-1)$ st level $x_{i} / x_{i-1}$ times before level $i$ has reached its capacity of $x_{i}$ candidate sets. To add $x_{i-1}$ candidate sets from level $i-1$ to level $i$, we need to reduce their sizes from $n / x_{i-1}^{d}$ to $n / x_{i}^{d}$. By Corollary 6 this requires at most $x_{i-1} d c\left(\log x_{i}-\log x_{i-1}\right) / \log x_{i-1}$ queries. The additional $x_{1} d \log x_{1}$ term accounts for the queries needed to move the sets from level 0 to level 1; i.e., for the randomized binary search algorithm through which we initially reduce the sizes of the $V_{i}$ s to $n / x_{1}^{d}$-requiring at most $d \log x_{1}$ queries per call. Finally, the term $x_{t} \log n / \log x_{t}$ accounts for the final reduction of the $V_{i}$ s to a set containing only one single element (at this stage we shall finally have $\left.V_{i}=\{\pi(i)\}\right)$. More precisely, this term is $\left(x_{t}\left(\log n-d \log x_{t}\right)\right) / \log x_{t}$ but we settle for upper bounding this expression by the term given in the formula.

Next we need to bound the number of queries caused by failures. We show that, on average, not too many failures happen. More precisely, we show that the expected number of level- $i$ failures is at most $n^{2} /\left((n-q)\left(x_{i}^{d-1}-1\right)\right)$. By Corollary 6 , each such level- $i$ failure causes an additional number of at most $1+x_{i} d c\left(\log x_{i+1}-\log x_{i}\right) / \log x_{i}$ queries (the 1 counts for the query through which we discover that $\left.\pi\left(s^{*}+1\right) \in V_{1} \cup \ldots \cup V_{s^{*}}\right)$. Thus,

$$
\sum_{i=1}^{t} \frac{n^{2}}{(n-q)\left(x_{i}^{d-1}-1\right)}\left(1+\frac{x_{i} d c\left(\log x_{i+1}-\log x_{i}\right)}{\log x_{i}}\right)
$$

bounds the expected number of additional queries caused by failures.

We recall the settings of the $x_{i}$. We have $x_{1}=\log n$ and $x_{i}=\log ^{2^{i-1}} n$. We further have $t \in \Theta(\log \log n)$, so that $\log x_{t}=\Omega(\log n)$. The parameter $d$ is some constant $\geq 4$. With these parameter settings, formula (1) evaluates to

$$
n d c\left(\frac{\log n}{\log x_{t}}+2(t-1)+\log \log n-t\right)=O(n \log \log n)
$$


and, somewhat wasteful, we can bound formula (2) from above by

$$
\begin{aligned}
\frac{n^{2} d c}{n-q} \sum_{i=1}^{t} x_{i}^{-(d-3)} & =O(n \log n) \sum_{i=0}^{t-1} x_{1}^{-(d-3) 2^{i}} \\
& <O(n \log n)\left(x_{1}^{d-3}-1\right)^{-1}=O(n) .
\end{aligned}
$$

This shows that the overall expected number of queries sums to $O(n \log \log n)+O(n)=$ $O(n \log \log n)$.

\section{The Lower Bound}

In this section, we prove a tight lower bound for the randomized query complexity of the HiddenPermutation problem. The lower bound is stated in the following:

Theorem 7. The randomized query complexity of the HidDEnPERMUTATION problem with $n$ positions is $\Omega(n \log \log n)$.

To prove a lower bound for randomized query schemes, we appeal to Yao's principle. That is, we first define a hard distribution over the secrets and show that every deterministic query scheme for this hard distribution needs $\Omega(n \log \log n)$ queries in expectation. This part of the proof is done using a potential function argument.

Hard Distribution Let $\Pi$ be a permutation drawn uniformly among all the permutations of $[n]$ (in this section, we use capital letters to denote random variables). Given such a permutation, we let our target string $Z$ be the one satisfying $Z_{\Pi(i)}=(i \bmod 2)$ for $i=1, \ldots, n$. Since $Z$ is uniquely determined by the permutation $\Pi$, we will mostly ignore the role of $Z$ in the rest of this section. Finally, we use $F(x)$ to denote the value of the random variable $f_{Z, \Pi}(x)$ for $x \in\{0,1\}^{n}$. We will also use the notation $a \equiv b$ to mean that $a \equiv b \bmod 2$.

Deterministic Query Schemes By fixing the random coins, a randomized solution with expected $t$ queries implies the existence of a deterministic query scheme with expected $t$ queries over our hard distribution. The rest of this section is devoted to lower bounding $t$ for such a deterministic query scheme.

A deterministic query scheme is a decision tree $T$ in which each node $v$ is labeled with a string $x_{v} \in\{0,1\}^{n}$. Each node has $n+1$ children, numbered from 0 to $n$, and the $i$ th child is traversed if $F\left(x_{v}\right)=i$. To guarantee correctness, no two inputs can end up in the same leaf.

For a node $v$ in the decision tree $T$, we define $\max _{v}$ as the largest value of $F$ seen along the edges from the root to $v$. Note that $\max _{v}$ is not a random variable and in fact, at any node $v$ and for any ancestor $u$ of $v$, conditioned on the event that the search path reaches $v$, the value of $F\left(x_{u}\right)$ is equal to the index of the child of $u$ that lies on the path to $v$. Finally, we define $S_{v}$ as the subset of inputs (as outlined above) that reach node $v$.

We use a potential function which measures how much "information" the queries asked have revealed about $\Pi$. Our goal is to show that the expected increase in the potential function after asking each query is small. Our potential function depends crucially on the candidate sets. The update rules for the candidate sets are slightly more specific than the ones in Section 2 because we now have a fixed connection between the two parts of the secret. We denote the candidate set for $\pi(i)$ at node $v$ with $V_{i}^{v}$. At the root node $r$, we have $V_{i}^{r}=[n]$ for all $i$. Let $v$ be a node in the tree and let $w_{0}, \ldots, w_{n}$ be its children $\left(w_{i}\right.$ is traversed when the score $i$ is returned). Let $P_{0}^{v}$ (resp. $P_{1}^{v}$ ) be the set of positions in $x_{v}$ that contain 0 (resp. 1). Thus, 
formally, $P_{0}^{v}=\left\{i \mid x_{v}[i]=0\right\}$ and $P_{1}^{v}=\left\{i \mid x_{v}[i]=1\right\} .{ }^{1}$ The precise definition of candidate sets is as follows:

$$
V_{i}^{w_{j}}= \begin{cases}V_{i}^{v} \cap P_{i \bmod 2}^{v} & \text { if } i \leq j, \\ V_{i}^{v} \cap P_{j \bmod 2}^{v} & \text { if } i=j+1, \\ V_{i}^{v} & \text { if } i>j+1 .\end{cases}
$$

As with the upper bound case, the candidate sets have some very useful properties. These properties are slightly different from the ones observed before, due to the fact that some extra information has been announced to the query algorithm. We say that a candidate set $V_{i}^{v}$ is active (at $v$ ) if the following conditions are met: (i) at some ancestor node $u$ of $v$, we have $F\left(x_{u}\right)=i-1$, (ii) at every ancestor node $w$ of $u$ we have $F\left(x_{w}\right)<i-1$, and (iii) $i<\min \left\{n / 3, \max _{v}\right\}$. We call $V_{\max _{v}+1}^{v}$ pseudo-active (at $v$ ).

For intuition on the requirement $i<n / 3$, observe from the following lemma that $V_{\max _{v}+1}^{v}$ contains all sets $V_{i}^{v}$ for $i \leq \max _{v}$ and $i \equiv \max _{v}$. At a high level, this means that the distribution of $\Pi\left(\max _{v}+1\right)$ is not independent of $\Pi(i)$ for $i \equiv \max _{v}$. The bound $i<n / 3$, however, forces the dependence to be rather small (there are not too many such sets). This greatly helps in the potential function analysis.

In the appendix, we show that the candidate sets satisfy the following:

Lemma 8. The candidate sets have the following properties:

(i) Two candidate sets $V_{i}^{v}$ and $V_{j}^{v}$ with $i<j \leq \max _{v}$ and $i \not \equiv j$ are disjoint.

(ii) An active candidate set $V_{j}^{v}$ is disjoint from any candidate set $V_{i}$ provided $i<j<\max _{v}$.

(iii) The candidate set $V_{i}^{v}, i \leq \max _{v}$ is contained in the set $V_{\max _{v}+1}^{v}$ if $i \equiv \max _{v}$ and is disjoint from it if $i \not \equiv \max _{v}$.

(iv) For two candidate sets $V_{i}^{v}$ and $V_{j}^{v}, i<j$, if $V_{i}^{v} \cap V_{j}^{v} \neq \emptyset$ then $V_{i}^{v} \subset V_{j}^{v}$.

\subsection{Potential Function}

We define the potential of an active candidate set $V_{i}^{v}$ as $\log \log \left(2 n /\left|V_{i}^{v}\right|\right)$. This is inspired by the upper bound: a potential increase of 1 corresponds to a candidate set advancing one level in the upper bound context (in the beginning, a set $V_{i}^{v}$ has size $n$ and thus its potential is 0 while at the end its potential is $\Theta(\log \log n)$. With each level, the quantity $n$ divided by the size of $V_{i}$ is squared). We define the potential at a node $v$ as

$$
\varphi(v)=\log \log \frac{2 n}{\left|V_{\max _{v}+1}^{v}\right|-\mathrm{Con}_{v}}+\sum_{j \in A_{v}} \log \log \frac{2 n}{\left|V_{j}^{v}\right|},
$$

in which $A_{v}$ is the set of indices of active candidate sets at $v$ and $\mathrm{Con}_{v}$ is the number of candidate sets contained inside $V_{\max _{v}+1}^{v}$. Note that from Lemma 8, it follows that $\operatorname{Con}_{v}=\left\lfloor\max _{v} / 2\right\rfloor$.

The intuition for including the term $\mathrm{Con}_{v}$ is the same as our requirement $i<n / 3$ in the definition of active candidate sets, namely that once $\operatorname{Con}_{v}$ approaches $\left|V_{\max _{v}+1}^{v}\right|$, the distribution of $\Pi\left(\max _{v}+1\right)$ starts depending heavily on the candidate sets $V_{i}^{v}$ for $i \leq \max _{v}$ and $i \equiv \max _{v}$. Thus we have in some sense determined $\Pi\left(\max _{v}+1\right)$ already when $\left|V_{\max _{v}+1}^{v}\right|$ approaches $\operatorname{Con}_{v}$. Therefore, we have to take this into account in the potential function since otherwise changing $V_{\max _{v}+1}^{v}$ from being pseudo-active to being active could give a huge potential increase.

After some lengthy calculations, it is possible to prove the following lemma. The full proof is presented in Appendix C. Here instead, we give only a very high level overview.

\footnotetext{
${ }^{1}$ To prevent our notations from becoming too overloaded, here and in the remainder of the section we write $x=(x[1], \ldots, x[n])$ instead of $x=\left(x_{1}, \ldots, x_{n}\right)$
} 
Lemma 9. Let $v$ be a node in $T$ and let $\boldsymbol{i}_{v}$ be the random variable giving the value of $F\left(x_{v}\right)$ when $\Pi \in S_{v}$ and 0 otherwise. Also let $w_{0}, \ldots, w_{n}$ denote the children of $v$, where $w_{j}$ is the child reached when $F\left(x_{v}\right)=j$. Then, $\mathbb{E}\left[\varphi\left(w_{i_{v}}\right)-\varphi(v) \mid \Pi \in S_{v}\right]=O(1)$.

Note that we have $\mathbb{E}\left[\varphi\left(w_{\mathbf{i}_{v}}\right)-\varphi(v) \mid \Pi \in S_{v}\right]=\sum_{a=0}^{n} \operatorname{Pr}\left[F\left(x_{v}\right)=a \mid \Pi \in S_{v}\right]\left(\varphi\left(w_{a}\right)-\varphi(v)\right)$. We consider two main cases: $F\left(x_{v}\right) \leq \max _{v}$ and $F\left(x_{v}\right)>\max _{v}$. In the first case, the maximum score will not increase in $w_{a}$ which means $w_{a}$ will have the same set of active candidate sets. In the second case, the pseudo-active candidate set $V_{\max _{v}+1}^{v}$ will turn into an active set $V_{\max _{v}+1}^{w_{a}}$ at $w_{a}$ and $w_{a}$ will have a new pseudo-active set. While this second case looks more complicated, it is in fact the less interesting part of the analysis. This is because the probability of suddenly increasing the score by $\alpha$ is extremely small (we will show that it is roughly $2^{-\Omega(\alpha)}$ ) which subsumes any significant potential increase for values of $a>\max _{v}$.

Let $a_{1}, \ldots, a_{\left|A_{v}\right|}$ be the indices of active candidate sets at $v$ sorted in increasing order. We also define $a_{\left|A_{v}\right|+1}=\max _{v}+1$. For a candidate set $V_{i}^{v}$, and a Boolean $b \in\{0,1\}$, let $V_{i}^{v}(b)=\left\{j \in V_{i}^{v} \mid x_{v}[j]=b\right\}$. Clearly, $\left|V_{i}^{v}(0)\right|+\left|V_{i}^{v}(1)\right|=\left|V_{i}^{v}\right|$. For even $a_{i}, 1 \leq i \leq\left|A_{v}\right|$, let

$$
\varepsilon_{i}=\left|V_{i}^{v}(1)\right| /\left|V_{i}^{v}\right|
$$

and for odd $i$, let

$$
\varepsilon_{i}=\left|V_{i}^{v}(0)\right| /\left|V_{i}^{v}\right|
$$

Thus $\varepsilon_{i}$ is the fraction of locations in $V_{i}^{v}$ that contains values that does not match $Z_{\Pi(i)}$. Also, we define

$$
\varepsilon_{i}^{\prime}:=\operatorname{Pr}\left[a_{i} \leq F\left(x_{v}\right)<a_{i+1}-1 \mid \Pi \in S_{v} \wedge F\left(x_{v}\right) \geq a_{i}\right] .
$$

Note that $\varepsilon_{i}^{\prime}=0$ if $a_{i+1}=a_{i}+1$.

With these definitions, it is clear that we have

$$
\begin{array}{r}
\left|V_{a_{i}}^{w_{j}}\right|=\left(1-\varepsilon_{i}\right)\left|V_{a_{i}}^{v}\right| \quad \text { for } \quad a_{i} \leq j . \\
\left|V_{a_{i}}^{w_{j}}\right|=\varepsilon_{i}\left|V_{a_{i}}^{v}\right| \text { for } \quad a_{i}=j+1 . \\
\left|V_{a_{i}}^{w_{j}}\right|=\left|V_{a_{i}}^{v}\right| \text { for } \quad a_{i}>j+1 .
\end{array}
$$

The important fact is that we can also bound other probabilities using the $\varepsilon_{i}$ s and $\varepsilon_{i}^{\prime}$ s: We can show that (see the appendix)

$$
\operatorname{Pr}\left[F\left(x_{v}\right)=a_{i}-1 \mid \Pi \in S_{v}\right] \leq \varepsilon_{i} \Pi_{j=1}^{i-1}\left(1-\varepsilon_{j}\right)\left(1-\varepsilon_{j}^{\prime}\right)
$$

and

$$
\operatorname{Pr}\left[a_{i} \leq F\left(x_{v}\right)<a_{i+1}-1 \mid \Pi \in S_{v}\right] \leq \varepsilon_{i}^{\prime}\left(1-\varepsilon_{i}\right) \Pi_{j=1}^{i-1}\left(1-\varepsilon_{j}\right)\left(1-\varepsilon_{j}^{\prime}\right) .
$$

Thus we have bounds on the changes in the size of the active candidate sets in terms of the $\varepsilon_{i} \mathrm{~s}$. The probability of making the various changes is also determined from the $\varepsilon_{i} \mathrm{~s}$ and $\varepsilon_{i}^{\prime} \mathrm{s}$ and finally the potential function is defined in terms of the sizes of these active candidate sets. Thus proving Lemma 9 reduces to proving an inequality showing that any possible choice of the $\varepsilon_{i} \mathrm{~S}$ and $\varepsilon_{i}^{\prime}$ s provide only little expected increase in potential.

Since the full calculations are too long to fit in this extended abstract, we will in the following provide intuition for the correctness by giving parts of the analysis for the seemingly best choice for the $\varepsilon_{i}$ s and simply assume all $\varepsilon_{i}^{\prime}$ s are 0 ; i.e., $a_{i}=i$ for all $i \leq \max _{v}$. Also, we ignore the term in $\varphi(v)$ involving $\mathrm{Con}_{v}$ and consider only the case where the score returned is no larger than $\max _{v}$ and $\max _{v}=n / 4$. 
From an information theoretic perspective, queries reveal the most information when the answer is uniform amongst many possibilities; i.e., if the entropy of the answer is high. Examining the probability bounds above, this corresponds to the setting where we essentially have $\varepsilon_{i}=\prod_{j=1}^{i-1}\left(1-\varepsilon_{j}\right)$. This is roughly satisfied for the choice $\varepsilon_{i}=1 / \max _{v}=4 / n$. Therefore, we will analyze the expected potential increase for the choice $\varepsilon_{i}=4 / n$ for all $i \leq n / 4$. For this choice, the above probability bound (3) becomes (using $\left.a_{i}=i\right) \operatorname{Pr}\left[F\left(x_{v}\right)=i-1 \mid \Pi \in S_{v}\right] \leq \Theta(1 / n)$. Thus the expected increase in potential provided by the cases where the score does not increase is bounded by

$$
\sum_{j \leq n / 4} \operatorname{Pr}\left[F\left(x_{v}\right)=j \mid \Pi \in S_{v}\right]\left(\varphi\left(w_{j}\right)-\varphi(v)\right) \leq \sum_{j \leq n / 4} \Theta\left(\frac{1}{n}\right)\left(\varphi\left(w_{j}\right)-\varphi(v)\right) .
$$

Also, we get that when a score of $j$ is returned, we update

$$
\begin{array}{r}
\left|V_{i}^{w_{j}}\right|=(1-\Theta(1 / n))\left|V_{i}^{v}\right| \text { for } \quad i \leq j . \\
\left|V_{i}^{w_{j}}\right|=\Theta\left(\left|V_{i}^{v}\right| / n\right) \text { for } i=j+1 . \\
\left|V_{i}^{w_{j}}\right|=\left|V_{i}^{v}\right| \text { for } \quad i>j+1 .
\end{array}
$$

Examining $\varphi\left(w_{j}\right)-\varphi(v)$ and the changes to the candidate sets, we get that there is one candidate set whose size decreases by a factor $n / 4$, and there are $j$ sets that change by a factor $(1-4 / n)$. Here we only consider the potential change caused by the sets changing by a factor of $n / 4$. This change is bounded by

$$
\sum_{j \leq n / 4} \Theta\left(\frac{1}{n}\right) \log \left(\frac{\log \left(n^{2} / 2\left|V_{j}^{v}\right|\right)}{\log \left(2 n /\left|V_{j}^{v}\right|\right)}\right)=\sum_{j \leq n / 4} \Theta\left(\frac{1}{n}\right) \log \left(1+\frac{\log (n / 4)}{\log \left(2 n /\left|V_{j}^{v}\right|\right)}\right) .
$$

To continue these calculations, we use Lemma 8 to conclude that the active candidate sets are disjoint and hence the sum of their sizes is bounded by $n$. We now divide the sum into summations over indices where $\left|V_{j}^{v}\right|$ is in the range $\left[2^{i}: 2^{i+1}\right]$ :

$$
\begin{aligned}
\sum_{j \leq n / 4} \Theta\left(\frac{1}{n}\right) \log \left(1+\frac{\log (n / 4)}{\log \left(2 n /\left|V_{j}^{v}\right|\right)}\right) & \leq \Theta\left(\sum_{i=0}^{\log n-1} \frac{1}{2^{i}} \log \left(1+\frac{\log (n / 4)}{\log \left(n / 2^{i}\right)}\right)\right) \\
& \leq \Theta\left(\sum_{i=0}^{\log n-1} \frac{\log (n / 4)}{2^{i} \log \left(n / 2^{i}\right)}\right) .
\end{aligned}
$$

The last inequality followed from $\log (1+x) \leq x$ for $x>0$. Now the sum over the terms where $i>\log \log n$ is clearly bounded by a constant since the $2^{i}$ in the denominator cancels the $\log (n / 4)$ term and we get a geometric series of this form. For $i<\log \log n$, we have $\log (n / 4) / \log \left(n / 2^{i}\right)=O(1)$ and we again have a geometric series summing to $O(1)$.

The full proof is very similar in spirit to the above, just significantly more involved due to the unknown values $\varepsilon_{i}$ and $\varepsilon_{i}^{\prime}$. For the complete proof see Appendix C.

\subsection{Potential at the End}

Intuitively, if the maximum score value increases after a query, it increases, in expectation, only by an additive constant. In fact, in Appendix $\mathrm{C}$, we prove that the probability of increasing 
the maximum score value by $\alpha$ after one query is $2^{-\Omega(\alpha)}$. Thus, it follows from the definition of the active candidate sets that when the score reaches $n / 3$ we expect $\Omega(n)$ active candidate sets. However, by Lemma 8 , the active candidate sets are disjoint. This means that a fraction of them (again at least $\Omega(n)$ of them), must be small, or equivalently, their total potential is $\Omega(n \log \log n)$. In the rest of this section, we prove this intuition.

Given an input $(z, \pi)$, we say an edge $e$ in the decision tree $T$ is increasing if $e$ corresponds to an increase in the maximum score and it is traversed given the input $(z, \pi)$. We say that an increasing edge is short if it corresponds to an increase of at most $c$ in the maximum function score (in which $c$ is a sufficiently large constant) and we call it long otherwise. Let $N$ be the random variable denoting the number of increasing edges seen on input $\Pi$ before reaching a node with score greater than $n / 3$. Let $L_{j}$ be the random indicator variable taking the value 0 if the $j$ th increasing edge is short, and taking the value equal to the amount of increase in the score along this edge if not. If $j>N$, then we define $L_{j}=0$. Also let $W_{j}$ be the random variable corresponding to the node of the decision tree where the $j$ th increase happens. As discussed, in Appendix $\mathrm{C}$ we prove that for every node $v, \operatorname{Pr}\left[L_{j} \geq \alpha \mid W_{j}=v\right] \leq 2^{-\Omega(\alpha)}$. We want to upper bound $\sum_{j=1}^{n} \mathbb{E}\left[L_{j}\right]$ (there are always at most $n$ increasing edges). From the above, we know that

$$
\begin{aligned}
\mathbb{E}\left[L_{j}\right] & \leq \mathbb{E}\left[L_{j} \mid N \geq j\right] \\
& =\sum_{v \in T} \sum_{i=c+1}^{n} i \cdot \operatorname{Pr}\left[L_{j}=i \wedge W_{j}=v \mid N \geq j\right] \\
& =\sum_{v \in T} \sum_{i=c+1}^{n} i \cdot \operatorname{Pr}\left[L_{j}=i \wedge W_{j}=v\right] \\
& =\sum_{v \in T} \sum_{i=c+1}^{n} i \cdot \operatorname{Pr}\left[L_{j}=i \mid W_{j}=v\right] \operatorname{Pr}\left[W_{j}=v\right] \\
& \leq \sum_{v \in T} \sum_{i=c+1}^{n} \frac{i}{2^{\Omega(i)}} \operatorname{Pr}\left[W_{j}=v\right] \\
& \leq \sum_{v \in T} \frac{1}{2^{\Omega(c)}} \operatorname{Pr}\left[W_{j}=v\right] \leq \frac{1}{2^{\Omega(c)}}
\end{aligned}
$$

where the summation is taken over all nodes $v$ in the decision tree $T$. The computation shows $\sum_{j=1}^{n} \mathbb{E}\left[L_{j}\right] \leq n / 2^{\Omega(c)}$. By Markov's inequality, we get that with probability at least $3 / 4$, we have $\sum_{j=1}^{n} L_{j} \leq n / 2^{\Omega(c)}$. Thus, when the function score reaches $n / 3$, short edges must account for $n / 3-n / 2^{\Omega(c)}$ of the increase which is at least $n / 6$ for a large enough constant $c$. Since any short edge has length at most $c$, there must be at least $n /(6 c)$ short edges. As discussed, this implies existence of $\Omega(n)$ active candidate sets that have size $O(1)$, meaning, their contribution to the potential function is $\Omega(\log \log n)$ each. We have thus shown:

Lemma 10. Let $\ell$ be the random variable giving the leaf node of $T$ that the deterministic query scheme ends up in on input $\Pi$. We have $\varphi(\ell)=\Omega(n \log \log n)$ with probability at least $3 / 4$.

\subsection{Putting Things Together}

Finally, we show how Lemma 9 and Lemma 10 combine to give our lower bound. Essentially this boils down to showing that if the query scheme is too efficient, then the query asked at some node of $T$ increases the potential by $\omega(1)$ in expectation, contradicting Lemma 9 . To show this 
explicitly, define $\mathbf{t}$ as the random variable giving the number of queries asked on input $\Pi$. We have $\mathbb{E}[\mathbf{t}]=t$, where $t$ was the expected number of queries needed for the deterministic query scheme. Also let $\ell_{1}, \ldots, \ell_{4 t}$ be the random variables giving the first $4 t$ nodes of $T$ traversed on input $\Pi$, where $\ell_{1}=r$ is the root node and $\ell_{i}$ denotes the node traversed at the $i$ th level of $T$. If only $m<4 t$ nodes are traversed, define $\ell_{i}=\ell_{m}$ for $i>m$; i.e., $\varphi\left(\ell_{i}\right)=\varphi\left(\ell_{m}\right)$. From Lemma 10, Markov's inequality and a union bound, we may now write

$$
\begin{aligned}
\mathbb{E}\left[\varphi\left(\ell_{4 t}\right)\right] & =\mathbb{E}\left[\varphi\left(\ell_{1}\right)+\sum_{i=1}^{4 t-1} \varphi\left(\ell_{i+1}\right)-\varphi\left(\ell_{i}\right)\right] \\
& =\mathbb{E}[\varphi(r)]+\mathbb{E}\left[\sum_{i=1}^{4 t-1} \varphi\left(\ell_{i+1}\right)-\varphi\left(\ell_{i}\right)\right] \\
& =\sum_{i=1}^{4 t-1} \mathbb{E}\left[\varphi\left(\ell_{i+1}\right)-\varphi\left(\ell_{i}\right)\right] \\
& =\Omega(n \log \log n) .
\end{aligned}
$$

Hence there exists a value $i^{*}$, where $1 \leq i^{*} \leq 4 t-1$, such that

$$
\mathbb{E}\left[\varphi\left(\ell_{i^{*}+1}\right)-\varphi\left(\ell_{i^{*}}\right)\right]=\Omega(n \log \log n / t) .
$$

But

$$
\mathbb{E}\left[\varphi\left(\ell_{i^{*}+1}\right)-\varphi\left(\ell_{i^{*}}\right)\right]=\sum_{v \in T_{i^{*}} \mid v \text { non-leaf }} \operatorname{Pr}\left[\Pi \in S_{v}\right] \mathbb{E}\left[\varphi\left(w_{\mathbf{i}_{v}}\right)-\varphi(v) \mid \Pi \in S_{v}\right],
$$

where $T_{i^{*}}$ is the set of all nodes at depth $i^{*}$ in $T, w_{0}, \ldots, w_{n}$ are the children of $v$ and $\mathbf{i}_{v}$ is the random variable giving the score of $F\left(x_{v}\right)$ on an input $\Pi \in S_{v}$ and 0 otherwise. Since the events $\Pi \in S_{v}$ and $\Pi \in S_{u}$ are disjoint for $v \neq u$, we conclude that there must exist a node $v \in T_{i^{*}}$ for which

$$
\mathbb{E}\left[\varphi\left(w_{\mathbf{i}_{v}}\right)-\varphi(v) \mid \Pi \in S_{v}\right]=\Omega(n \log \log n / t) .
$$

Combined with Lemma 9 this shows that $n \log \log n / t=O(1)$; i.e., $t=\Omega(n \log \log n)$. This concludes the proof of Theorem 7 .

\section{References}

[BBM12] Eric Blais, Joshua Brody, and Kevin Matulef, Property testing lower bounds via communication complexity, Computational Complexity 21 (2012), 311-358.

[BdW02] Harry Buhrman and Ronald de Wolf, Complexity measures and decision tree complexity: a survey, Theoretical Computer Science 288 (2002), 21-43.

[BGSMdW12] Harry Buhrman, David García-Soriano, Arie Matsliah, and Ronald de Wolf, The non-adaptive query complexity of testing $k$-parities, CoRR abs/1209.3849 (2012), Available at http://arxiv.org/abs/1209.3849.

[Bsh09] Nader H. Bshouty, Optimal algorithms for the coin weighing problem with a spring scale, Proc. of the 22nd Conference on Learning Theory (COLT'09), 2009.

[Chv83] Vasek Chvátal, Mastermind, Combinatorica 3 (1983), 325-329. 
[CK07] Amit Chakrabarti and Subhash Khot, Improved lower bounds on the randomized complexity of graph properties, Random Struct. Algorithms 30 (2007), 427-440.

[CM66] David G. Cantor and William H. Mills, Determination of a subset from certain combinatorial properties, Canadian Journal of Mathematics 18 (1966), 42-48.

[DJW02] Stefan Droste, Thomas Jansen, and Ingo Wegener, On the analysis of the $(1+1)$ evolutionary algorithm, Theoretical Computer Science 276 (2002), 51-81.

[DJW06] Stefan Droste, Thomas Jansen, and Ingo Wegener, Upper and lower bounds for randomized search heuristics in black-box optimization, Theory of Computing Systems 39 (2006), 525-544.

[DSTW13] Benjamin Doerr, Reto Spöhel, Henning Thomas, and Carola Winzen, Playing Mastermind with many colors, Proc. of the Twenty-Fourth Annual ACM-SIAM Symposium on Discrete Algorithms (SODA'13), SIAM, 2013, To appear. Available at http://arxiv.org/abs/1207.0773.

[DW12] Benjamin Doerr and Carola Winzen, Black-box complexity: Breaking the $O(n \log n)$ barrier of LeadingOnes, Proc. of Artificial Evolution (EA'11), Lecture Notes in Computer Science, vol. 7401, Springer, 2012, To appear. Available online at http://arxiv.org/abs/1210.6465.

[ER63] Paul Erdős and Alfréd Rényi, On two problems of information theory, Magyar Tudományos Akadémia Matematikai Kutató Intézet Közleményei 8 (1963), 229243.

[JKS03] T. S. Jayram, Ravi Kumar, and D. Sivakumar, Two applications of information complexity, Proceedings of the 35th Annual ACM Symposium on Theory of Computing (STOC'03), ACM, 2003, pp. 673-682.

[Lin65] Bernt Lindström, On a combinatorial problem in number theory, Canadian Mathematical Bulletin 8 (1965), 477-490.

[MNSX11] Frédéric Magniez, Ashwin Nayak, Miklos Santha, and David Xiao, Improved bounds for the randomized decision tree complexity of recursive majority, Proccedings of the 38th International Colloquium on Automata, Languages and Programming (ICALP'11), Lecture Notes in Computer Science, vol. 6755, Springer, 2011, pp. 317-329.

[Rud97] Günter Rudolph, Convergence properties of evolutionary algorithms, Kovac, 1997.

[SW86] Michael E. Saks and Avi Wigderson, Probabilistic boolean decision trees and the complexity of evaluating game trees, Proc. of the 27th Annual Symposium on Foundations of Computer Science (FOCS'86), IEEE Computer Society, 1986, pp. 29-38.

[Yao77] Andrew Chi-Chin Yao, Probabilistic computations: Toward a unified measure of complexity, Proc. of Foundations of Computer Science (FOCS'77), IEEE, 1977, pp. 222-227. 


\section{Appendix}

\section{A Details for Section 2}

We repeat Theorem 2 .

Theorem 2. It is decidable in polynomial time whether a guessing history $\mathcal{H}=\left(x^{i}, s^{i}\right)_{i=1}^{t}$ is feasible. Furthermore, we can efficiently compute the number of consistent pairs $(z, \pi) \in\{0,1\}^{n} \times S_{n}$.

We first show how to check feasibility in polynomial time.

of Theorem 2, Part I. We prove here the first part of Theorem 2; i.e., we show that consistency can be checked in polynomial time.

Let $\mathcal{H}=\left(x^{i}, s^{i}\right)_{i=1}^{t}$ be given. Construct the sets $V_{1}, \ldots, V_{n}$ as described in Theorem 1. Now construct a bipartite graph $G\left(V_{1}, \ldots, V_{n}\right)$ with node set $[n]$ on both sides. Connect $j$ to all nodes in $V_{j}$ on the other side. Permutations $\pi$ with $\pi(j) \in V_{j}$ for all $j$ are in one-to-one correspondence to perfect matchings in this graph. If there is no perfect matching, the history in infeasible. Otherwise, let $\pi$ be any permutation with $\pi(j) \in V_{j}$ for all $j$. We next construct $z$. We use the obvious rules:

a. If $i=\pi(j)$ and $j \leq s^{h}$ for some $h \in[t]$ then set $z_{i}:=x_{i}^{h}$.

b. If $i=\pi(j)$ and $j=s^{h}+1$ for some $h \in[t]$ then set $z_{i}:=1-x_{i}^{h}$.

c. If $z_{i}$ is not defined by one of the rules above, set it to an arbitrary value.

We need to show that these rules do not lead to a contradiction. Assume otherwise. There are three ways, in which we could get into a contradiction. There is some $i \in[n]$ and some $x^{h}, x^{\ell} \in\{0,1\}^{n}$

- setting $z_{i}$ to opposite values by rule (a)

- $\operatorname{setting} z_{i}$ to opposite values by rule (b)

- setting $z_{i}$ to opposite values by rules (b) applied to $x^{h}$ and rule (a) applied to $x^{\ell}$.

In each case, we readily derive a contradiction. In the first case, we have $j \leq s^{h}, j \leq s^{\ell}$ and $x_{i}^{h} \neq x_{i}^{\ell}$. Thus $\pi(j)=i \notin V_{j}$ by rule (1). In the second case, we have $j=s^{h}+1=s^{\ell}+1$ and $x_{i}^{h} \neq x_{i}^{\ell}$. Thus $i \notin V_{j}$ by (2). In the third case, we have $j=s^{h}+1, j \leq s^{\ell}$, and $x_{i}^{h}=x_{i}^{\ell}$. Thus $i \notin V_{j}$ by (3).

Finally, the pair $(z, \pi)$ defined in this way is clearly consistent with the history.

Next we show how to efficiently compute the number of consistent pairs. We recall Hall's condition for the existence of a perfect matching in a bipartite graph. A perfect matching exists if and only if $\left|\cup_{j \in J} V_{j}\right| \geq|J|$ for every $J \subseteq[n]$.

of Theorem 2, Part II. We now prove the second part of Theorem 2; i.e., we show how to efficiently count the number of consistent pairs.

According to Theorem 1 , the guessing history $\mathcal{H}$ can be equivalently described by a state $\left(V_{1}, \ldots, V_{s^{*}+1}, x^{*}, s^{*}\right)$. How many pairs $(z, \pi)$ are compatible with this state?

Once we have chosen $\pi$, there are exactly $2^{n-\left(s^{*}+1\right)}$ different choices for $z$ if $s^{*}<n$ and exactly one choice if $s^{*}=n$. The permutations can be chosen in a greedy fashion. We fix $\pi(1), \ldots, \pi(n)$ in this order. When we choose $\pi(i)$, the number of choices left is $\left|V_{i}\right|$ minus the 
number of $\pi(j), j<i$, lying in $V_{i}$. If $V_{j}$ is disjoint from $V_{i}, \pi(j)$ never lies in $V_{i}$ and if $V_{j}$ is contained in $V_{i}, \pi(j)$ is always contained in $V_{i}$. Thus the number of permutations is equal to

$$
\prod_{1 \leq i \leq n}\left(\left|V_{i}\right|-\left|\left\{j<i \mid V_{j} \subseteq V_{i}\right\}\right|\right) .
$$

It is easy to see that the greedy strategy does not violate Hall's condition.

The proof of Theorem 2 explains which values in $V_{i}$ are actually possible as value for $\pi(i)$. A value $\ell \in V_{i}$ is feasible if there is a perfect matching in the graph $G\left(V_{1}, \ldots, V_{n}\right)$ containing the edge $(i, \ell)$. The existence of such a matching can be decided in polynomial time; we only need to test for a perfect matching in the graph $G \backslash\{i, \ell\}$. Hall's condition says that there is no such perfect matching if there is a set $J \subseteq[n] \backslash\{i\}$ such that $\left|\cup_{j \in J} V_{j} \backslash\{\ell\}\right|<|J|$. Since $G$ contains a perfect matching (assuming a consistent history), this implies $\left|\cup_{j \in J} V_{j}\right|=|J|$; i.e., $J$ is tight for Hall's condition. We have shown: Let $\ell \in V_{i}$. Then $\ell$ is infeasible for $\pi(i)$ if and only if there is a tight set $J$ with $i \notin J$ and $\ell \in \cup_{j \in J} V_{j}$. Since the $V_{i}$ form a laminar family, minimal tight sets have a special form; they consist of an $i$ and all $j$ such that $V_{j}$ is contained in $V_{i}$. In the counting formula for the number of permutations such $i$ are characterized by $\left|V_{i}\right|-\left|\left\{j<i \mid V_{j} \subseteq V_{i}\right\}\right|=1$. In this situation, the elements of $V_{i}$ are infeasible for all $\pi(j)$ with $j>i$. We may subtract $V_{i}$ from each $V_{j}$ with $j>i$.

If Hall's condition is tight for some $J$, i.e., $\left|\cup_{j \in J} V_{j}\right|=|J|$, we can learn $\pi \mid J$ easily. We have $V_{j}=[n]$ for $j>s^{*}+1$ and hence the largest index in $J$ is at most $s^{*}+1$. Perturb $x^{*}$ by flipping each bit in $\cup_{j \in J} V_{j}$ exactly once. The objective values determine the permutation.

\section{B Details on the Randomized Strategy (Section 4)}

For readability purposes, we repeat the material presented in the main text. The reader only interested in the proof details may skip Section B.1 and most parts of Section B.4.

We repeat the main theorem of this section.

Theorem 4. The randomized query complexity of the HidDENPERMUTATION problem with $n$ positions is $O(n \log \log n)$.

The strategy verifying this bound has two parts. In the beginning, we use Algorithm 1 to identify the positions $\pi(1), \ldots, \pi(q)$ and the corresponding bit values $z_{\pi(1)}, \ldots, z_{\pi(q)}$; for some $q \in n-\Theta(n / \log n)$. As we shall see below, this requires $O(n \log \log n)$ queries, cf. Theorem 6 . Once $z_{\pi(1)}, \ldots, z_{\pi(q)}$ have been identified, we can find the remaining $n-q \in \Theta(n / \log n)$ positions and entries using the binary search algorithm described in the introduction (details will be given in Section B.2. This is the second phase of our algorithm. As the binary search strategy requires at most $O(\log n)$ queries per position, the second phase contributes only a linear number of queries - an effort that is negligible compared to the one of the first phase.

\section{B.1 Outline of the Proof for Theorem 4}

We remind the reader that the set $V_{i}$ is the candidate set for $\pi(i), 1 \leq i \leq n$. That is, we have $j \in V_{i}$ if - based on the query history so far - we have not ruled out that $\pi(i)$ equals $j$.

As noted in the main text, there is a trade-off between increasing the current best score $s^{*}$ and reducing the sizes of the candidate sets $V_{1}, \ldots, V_{s^{*}+1}$. The main idea behind Algorithm 1 is to alternate between the two. More precisely, we maintain a string $x$ and an integer $s$ with $f(x) \geq s$ and have already reduced some elements from $V_{1}$ to $V_{s}$. The sets $V_{s+1}$ to $V_{n}$ are still equal to [n]. Initially, $s=0$ and $x$ is arbitrary. The sets $V_{1}$ to $V_{s}$ are arranged into $t+2$ levels. 


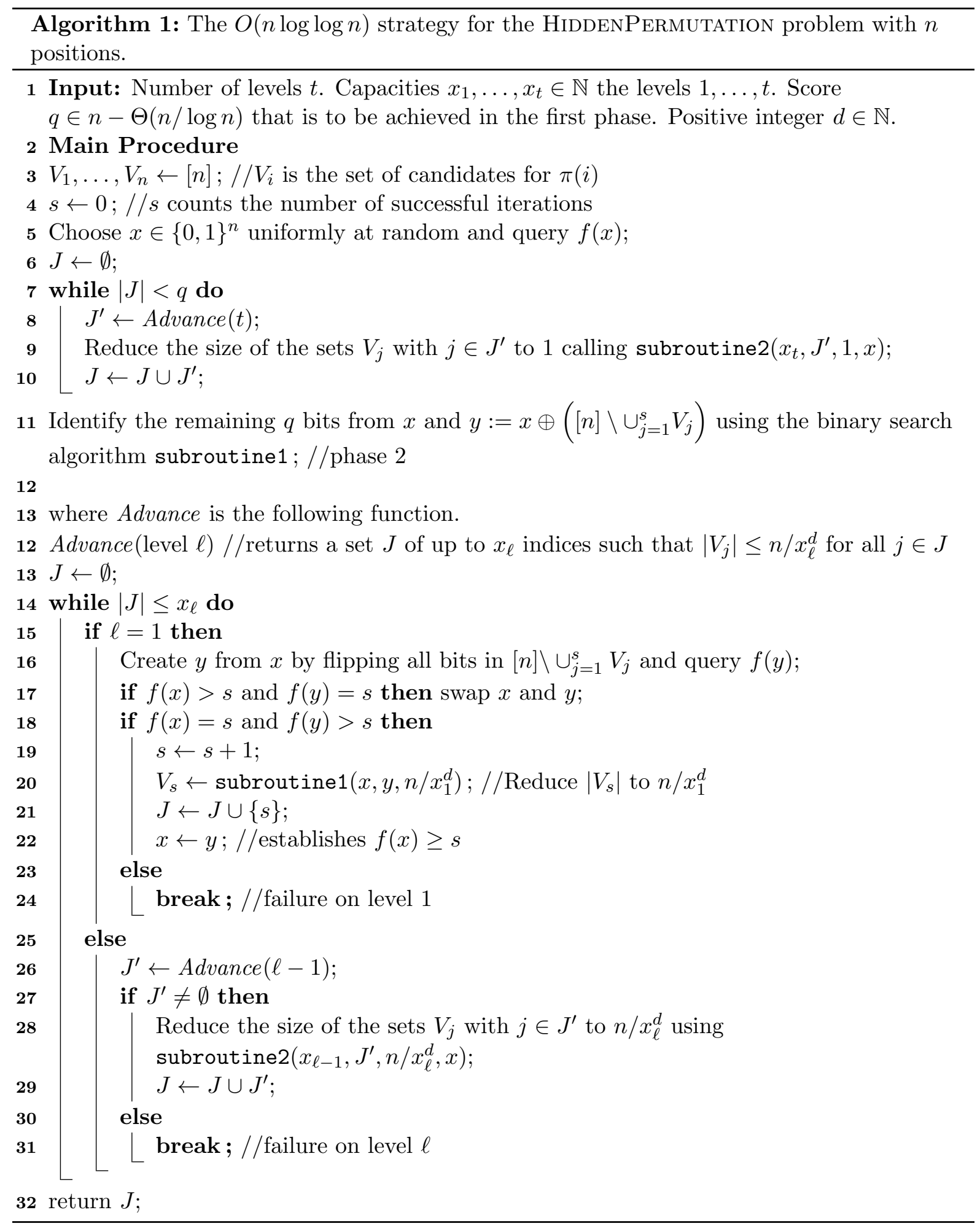

Initially, all candidate sets are on level 0 , and we have $V_{i}=[n]$ for all $i \in[n]$. The sets in level $i$ have larger index than the sets in level $i+1$. Level $t+1$ contains an initial segment of candidate sets; all candidate sets on level $t+1$ are singletons; i.e., we have identified the corresponding $\pi(i)$ value. On level $i, 1 \leq i \leq t$, we can have up to $x_{i}$ sets. We also say that the capacity of level $i$ is $x_{i}$. The size of any set on level $i$ is at most $n / x_{i}^{d}$, where $d \geq 1$ is some parameter to 
be fixed later. Once $x_{i}$ sets have been added to the $i$ th level, we reduce the size of each of them to at most $n / x_{i+1}^{d}$ using subroutine2, which we describe in Section B.3. The reduced sets are added to the $(i+1)$ st level. subroutine2 essentially allows us to simultaneously reduce the sizes of $k$ sets to a $k$ th fraction of their original size using at most $O(k)$ queries. Successive calls to subroutine 2 will reduce the sizes from at most $n / x_{i}^{d}$ to at most $n / x_{i+1}^{d}$. After moving the sets from level $i$ to level $i+1$, we either start filling the $i$ th level again (in case the $(i+1)$ st level has some capacity left), or we reduce the sizes of the sets on the $(i+1)$ st level, thus adding them to the $(i+2)$ nd one. Once the $t$ th level contains $x_{t}$ sets, we reduce the size of each of these sets to one, again employing subroutine2, and move them to level $t+1$. Thus $V_{j}=\{\pi(j)\}$ for each set $V_{j}$ on level $t+1$.

At level one (calls Advance(1)) we attempt to advance the score $s$. Let $s^{*}$ denote the current value of $s$ and let $x^{*}$ be the current bit string $x$. We ensure $f\left(x^{*}\right) \geq s^{*}$. We want to start working on $V_{s^{*}+1}$. We hope (this hope will be true with sufficiently high probability, as the analysis shows) that $\pi\left(s^{*}+1\right) \notin \cup_{j \leq s^{*}} V_{j}$. We create from $x^{*}$ a new bit string $y$ by flipping in $x^{*}$ all bits that are known not to be the image of $1, \ldots, s^{*}$ under $\pi$. That is, we set $y:=x^{*} \oplus\left([n] \backslash \cup_{j=1}^{s^{*}} V_{j}\right)$ and query $f(y)$. If $f(y)=s^{*}$ and $f(x)>s^{*}$, we swap the two strings. This step is needed to ensure correctness of subroutine1.

If (after the potential swapping of the names) $f(y)>s^{*}$ and $f\left(x^{*}\right)=s$ holds, we know that $\pi\left(s^{*}+1\right) \in[n] \backslash \cup_{j=1}^{s^{*}} V_{j}$. In this case, we immediately update $V_{s^{*}+1} \leftarrow[n] \backslash \cup_{j=1}^{s^{*}} V_{j}$ and we reduce the size of the set $V_{s^{*}+1}$ to $n / x_{1}^{d}$. This can be done by the binary search like algorithm, subroutine1, in at most $d \log x_{1}$ queries. This subroutine is described in Section B.2.

If, on the other hand, a failure happens; i.e., if in line $18 f(y)=f\left(x^{*}\right)=s^{*}$ or $f\left(x^{*}\right)>s^{*}$ holds, then we know that $\pi\left(s^{*}+1\right)$ has not been flipped. Hence $\pi\left(s^{*}+1\right) \in \cup_{j=1}^{s^{*}} V_{j}$ must hold. Therefore, we must reduce the size of $\cup_{j=1}^{s^{*}} V_{j}$ to "free" $\pi\left(s^{*}+1\right)$. We immediately abort the first level by reducing the size of each of the $V_{i}$ s currently on that level to $n / x_{2}^{d}$, thus adding them to the second level. Should we still not make progress by flipping all bits in $[n] \backslash \cup_{j=1}^{s^{*}} V_{j}$, we continue by reducing the size of each level-2 set to $n / x_{3}^{d}$, and so on, until we eventually have $\pi\left(s^{*}+1\right) \notin \cup_{j=1}^{s^{*}} V_{j}$, in which case we can continue with the "increase the score by one, then reduce the size of the candidate sets"-procedure described above.

As such failures cause queries that neither help us to advance $s^{*}$ nor to reduce the sizes of the candidate sets, it is essential to design the levels in such a way that the probability for such failures is small. As we shall see below, we achieve this by designing the levels such that their capacities grow exponentially. More precisely, we set $x_{i+1}=x_{i}^{2}$ for all $i=1, \ldots, t-1$. The first level has a capacity of $x_{1}=\log n$ sets. Thus, $t=O(\log \log n)$ levels are sufficient if we want the last level, level $t$, to have a capacity that is linear in $n$. Our choice of the $x_{i}$ s also guarantees that $x_{i}$ divides $x_{i+1}$ and hence a call Advance $(\ell)$ returns no more than $x_{\ell}$ elements (exactly $x_{\ell}$ elements if the call ends without a failure).

Our strategy is formalized by Algorithm 1. In what follows, we first present the two subroutines, subroutine1 and subroutine2. In Section B.4, we present the full proof of Theorem 4.

\section{B.2 Subroutine 1}

subroutine1 is called by the function Advance(1). Simulating a randomized binary search, it allows us to reduce the number of potential candidates for $\pi(i)$ from some value $v<n$ to some value $\ell<v$ in $\log v-\log \ell$ queries.

Lemma 11. Let $x, y \in\{0,1\}^{n}$ with $f(x)<f(y)$. Set $V:=\left\{j \in[n] \mid x_{j} \neq y_{j}\right\}$ and $v:=|V|$. Let $\ell \in \mathbb{N}, \ell<v$. Algorithm 2 reduces the size of $V$ to $\ell$ using at most $\lceil\log v-\log \ell\rceil$ queries. 


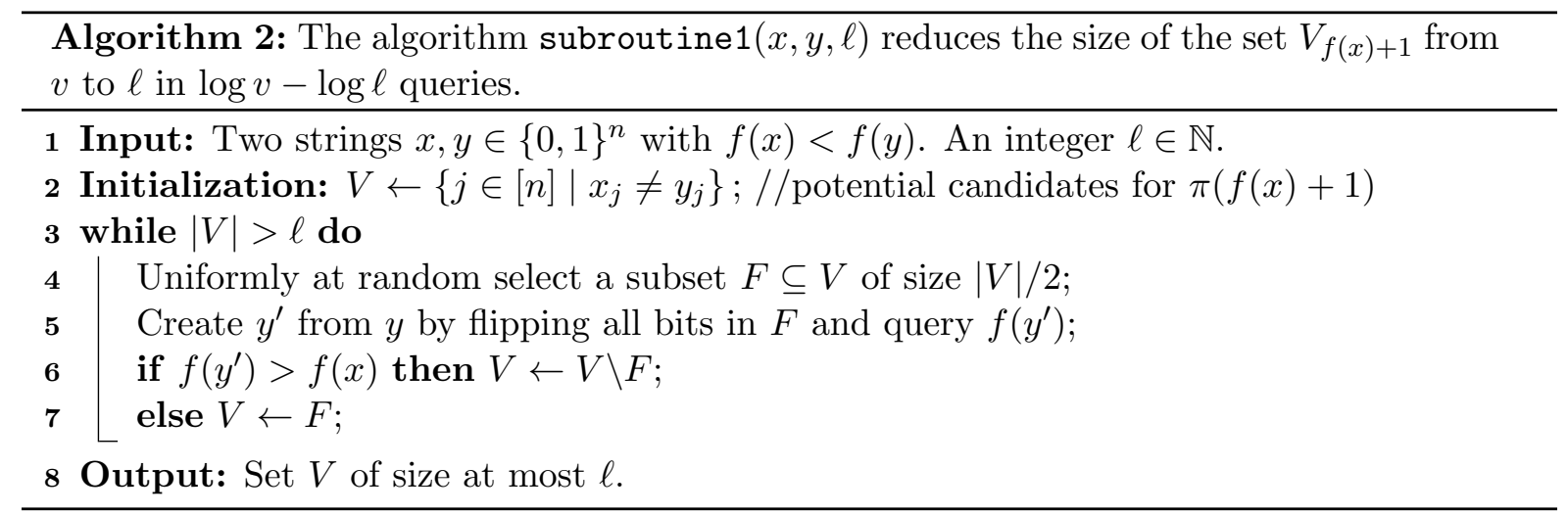

Proof. For the correctness of the algorithm we note that, by definition, we have $x_{\pi(i)}=y_{\pi(i)}=$ $z_{\pi(i)}$ for all $i \in[f(x)]$. Therefore, either we have $f\left(y^{\prime}\right)>f(x)$ in line 5 or we have $f\left(y^{\prime}\right)=f(x)$. In the former case, the bit $y_{\pi(f(x)+1)}$ was not flipped, and hence $\pi(f(x)+1) \notin F$ must hold. In the latter case the bit in position $\pi(f(x)+1)$ bit must have been flipped and we can infer $\pi(f(x)+1) \in F$.

The runtime bound is easily verified using the fact that the size of the set $V$ halves in each iteration.

It is now obvious how subroutine1 (with the "select uniformly at random select"-statement in line 4 replaced by "arbitrarily select") yields a deterministic $O(n \log n)$ query algorithm for the HiddenPermutation problem. This is Algorithm 3.

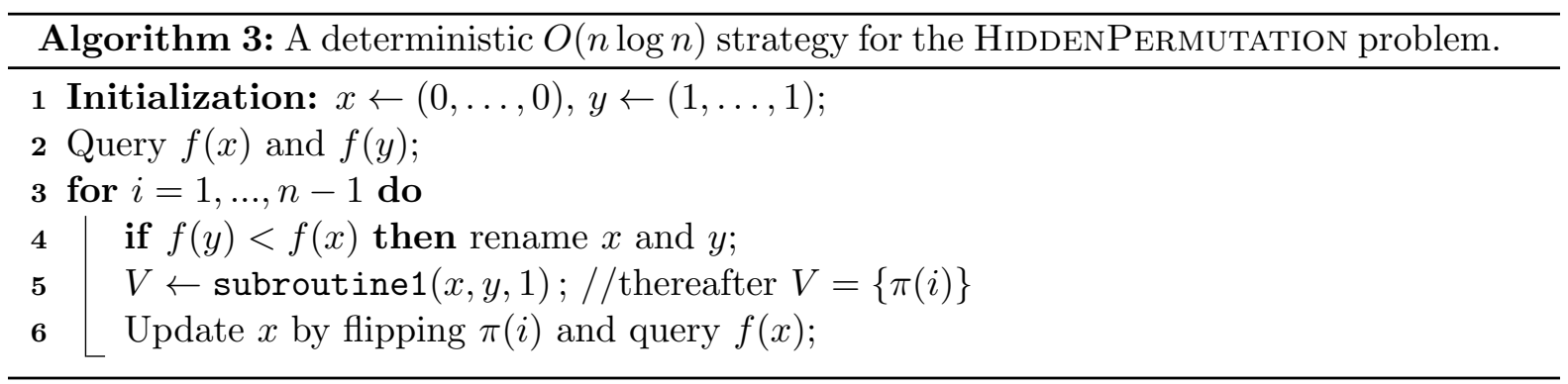

For the correctness of this algorithm, observe that after the execution of the $i$ th for-loop of Algorithm 3, the two strings $x$ and $y$ agree only in the positions $\pi(1), \ldots, \pi(i)$. Hence, either we have $f(x)=i$ and $f(y)>i$ or we have $f(y)=i$ and $f(x)>i$. In the latter case, $x$ and $y$ will be swapped in the next execution of the for-loop.

Note also that we apply this algorithm in the second phase of Algorithm 1 for identifying the last $\Theta(n / \log n)$ entries of $z$. This can be done as follows. When we leave the first phase of Algorithm 1, we have $\left|V_{1}\right|=\ldots=\left|V_{q}\right|=1$ and $f(x) \geq q$. Create $y$ from $x$ by flipping all bits in $[n] \backslash \cup_{i=1}^{q} V_{i}$ and query $f(y)$. Then jump into the for-loop of Algorithm 3. This shows how to determine the remaining $q \in \Theta(n / \log n)$ bits of the target string $z$ in at most a linear number of additional queries.

As mentioned, we call subroutine1 also in the first level of Algorithm 1 (line 20) to reduce the size of $V_{f(x)+1}$ to $n / x_{1}^{d}$, or, put differently, to reduce the number of candidates for $\pi(f(x)+1)$ to $n / x_{1}^{d}$. As the initial size of $V_{f(x)+1}$ is at most $n$, this requires at most $d \log x_{1}$ queries by Lemma 11. 


\section{B.3 Subroutine 2}

We describe the second subroutine of Algorithm 1, subroutine2. This routine is used to reduce the sizes of the up to $x_{\ell-1}$ candidate sets returned by a recursive call Advance $(\ell-1)$ from some value $\leq n / x_{\ell-1}^{d}$ to at most the target size of level $\ell$, which is $n / x_{\ell}^{d}$. As we shall see below, this requires an expected number of $O(1) x_{\ell-1} d\left(\log x_{\ell}-\log x_{\ell-1}\right) / \log x_{\ell-1}$ queries. The pseudo-code of subroutine2 is given in Algorithm 4. subroutine2 performs a subtask of this reduction: It reduces the sizes of at most $k$ candidate sets to a $k$ th fraction of their original size using at most $O(k)$ queries, where $k$ is a parameter. We use subroutine2 with parameter $k=x_{\ell-1}$ repeatedly to achieve the full reduction.

subroutine 2 is given a set $J$ of at most $k$ indices and a string $y$ with $f(y) \geq \max J$. The goal is to reduce the size of each candidate set $V_{j}, j \in J$, below a target size $m$ where $m \geq\left|V_{j}\right| / k$ for all $j \in J$. The routine works in phases, of several iterations each. Let $J$ be the set of indices of the candidate sets that are still above the target size at the beginning of an iteration. For each $j \in J$, we randomly choose a $k$ th fraction of the potential candidates for $\pi(j)$. That is, we randomly choose a subset $F_{j} \subseteq V_{j}$ of size $\left|V_{j}\right| / k$. We create a new bit string $y^{\prime}$ from $y$ by flipping all candidate positions $\cup_{j \in J} F_{j}$. A condition on the sets $\left(V_{j}\right)_{j \in J}$ ensures that we have either $f\left(y^{\prime}\right) \geq \max J$ or $f\left(y^{\prime}\right)=j-1$ for some $j \in J$.

In the first case, i.e., if $f\left(y^{\prime}\right) \geq \max J$, none of the sets $V_{j}$ was hit, and for all $j \in J$ we can remove the subset $F_{j}$ from $V_{j}$ as the elements in $F_{j}$ are no longer candidates for $\pi(j)$. We call such queries "off-trials". An off-trial reduces the size of all sets $V_{j}, j \in J$, to a $(1-1 / k)$ th fraction of their original size.

If, on the other hand, we have $f\left(y^{\prime}\right)=j-1$ for some $j \in J$, we can replace the candidate set $V_{j}$ by the set $F_{j}$ as $\pi(j) \in F_{j}$ must hold. Since $\left|F_{j}\right|=\left|V_{j}\right| / k \leq m$ by assumption, this set has now been reduced to its target size and we can remove it from $J$. We further know that for all $h \in J$ with $h<j$ the set $V_{h}$ was not hit. Thus, we can safely remove $F_{h}$ from $V_{h}$, for $h<j$.

We continue in this way until at least half of the indices are removed from $J$ and at least $c k$ off-trials occurred, for some constant $c$ satisfying $(1-1 / k)^{c k} \leq 1 / 2$. Once this is achieved, we are done with the current phase, and we move on to the next phase. To this end, let us consider any $j$ that is still in $J$. The size of $V_{j}$ was reduced by a factor $(1-1 / k)$ at least $c k$ times. Thus its size was reduced to at most half its original size. We may thus halve $k$ without destroying the invariant $m \geq\left|V_{j}\right| / k$ for $j \in J$. The effect of halving $k$ is that the relative size of the sets $F_{j}$ will be doubled for the sets $V_{j}$ that still take part in the reduction process.

We repeat Lemma 5.

Lemma 4. Let $k \in \mathbb{N}$, let $J \subseteq[n]$ be a set of at most $k$ indices with $V_{j} \cap V_{p}=\emptyset$ for $j \in J$ and $p \in[\max J] \backslash\{j\}$. Let $y \in\{0,1\}^{n}$ be such that $f(y) \geq \max J$, and let $m \in \mathbb{N}$ be such that $m \geq\left|V_{j}\right| / k$ for all $j \in J$.

In expectation it takes $O(k)$ queries until Algorithm 4 has reduced the size of $V_{j}$ to at most $m$ for each $j \in J$.

Proof. Let $c$ be some constant. We show below that-for a suitable choice of $c$-after an expected number of at most $c k$ queries both conditions in line 16 are satisfied. Assuming this to hold, we can bound the total expected number of queries until the size of each of the $V_{j}$ s has been reduced to $m$ by

$$
\sum_{h=0}^{\log k} c k / 2^{h}<2 c k,
$$

as desired. 


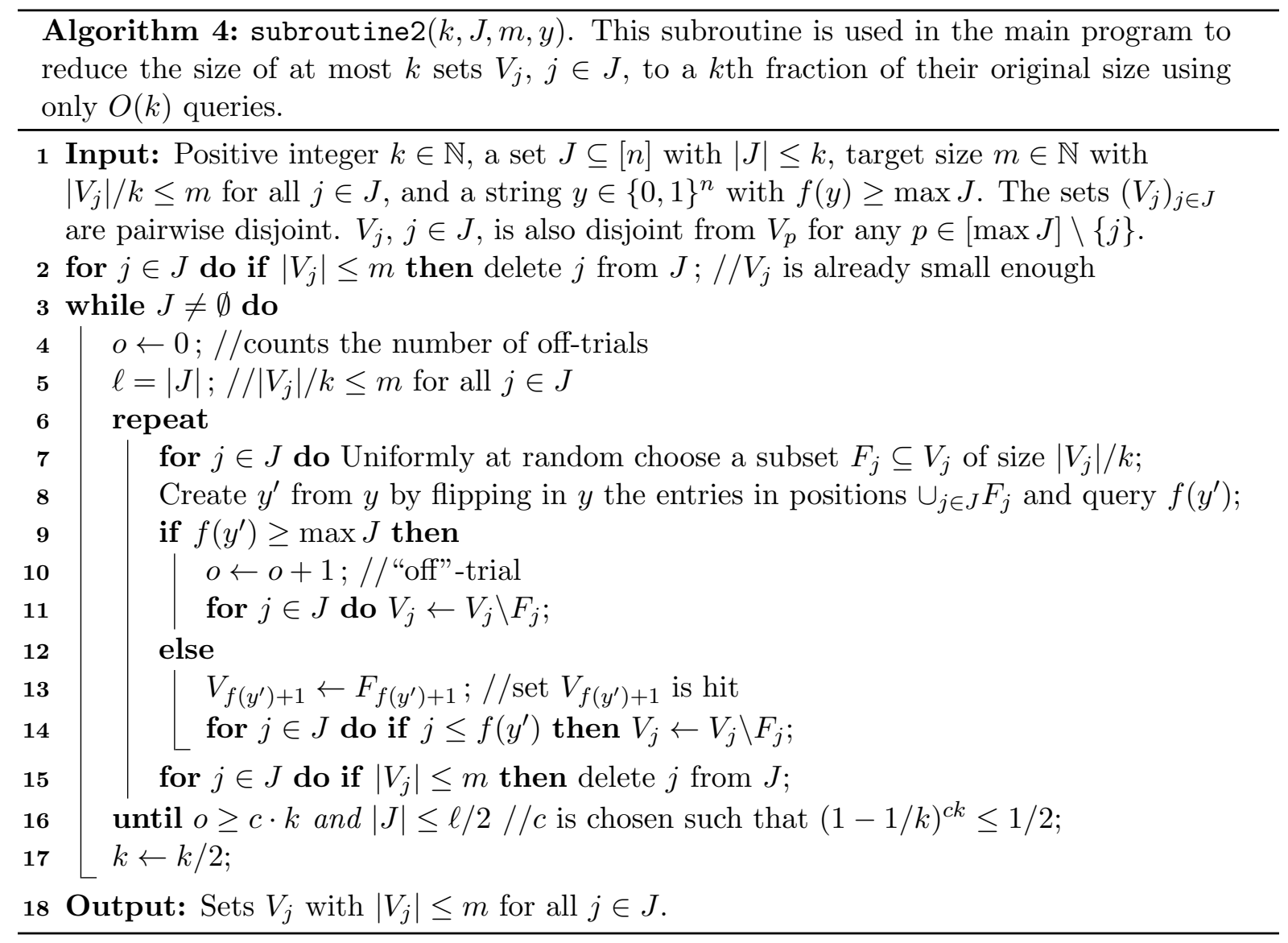

In each iteration of the repeat-loop we either hit an index in $J$ and hence remove it from $J$ or we have an off-trial. The probability of an off-trial is at least $(1-1 / k)^{k}$ since $|J| \leq k$ always. Thus the probability of an off-trial is at least $(2 e)^{-1}$ and hence the condition $o \geq c k$ holds after an expected number of $O(k)$ iterations.

As long as $|J| \geq \ell / 2$, the probability of an off-trial is at most $(1-1 / k)^{\ell / 2}$ and hence the probability that a set is hit is at least $1-(1-1 / k)^{\ell / 2}$. Since $\ln (1-1 / k) \leq-1 / k$ we have $(1-1 / k)^{\ell / 2}=\exp (\ell / 2 \ln (1-1 / k)) \leq \exp (-\ell /(2 k))$ and hence $1-(1-1 / k)^{\ell / 2} \geq 1-$ $\exp (-\ell /(2 k)) \geq \ell /(2 k)$. Thus the expected number of iterations to achieve $\ell / 2$ hits is $O(k)$.

If a candidate set $V_{j}$ is hit in the repeat-loop, its size is reduced to $\left|V_{j}\right| / k$. By assumption, this is bounded by $m$. If $V_{j}$ is never hit, its size is reduced at least $c k$ times by a factor $(1-1 / k)$. By choice of $c$, this is at most half of its original size. Thus after replacing $k$ by $k / 2$ we still have $\left|V_{j}\right| / k \leq m$ for $j \in J$.

Corollary 5. Let $k \in \mathbb{N}, J$, and $y$ be as in Lemma 4. Let further $d \in \mathbb{N}$ and $x \in \mathbb{R}$ such that $\max _{j \in J}\left|V_{j}\right|=n / x^{d}$. Let $y \in \mathbb{R}$ with $y>x$.

Using at most $d(\log y-\log x) / \log k$ calls to Algorithm 4 we can reduce the maximal size $\max _{j \in J}\left|V_{j}\right|$ to $n / y^{d}$. The overall expected number of queries needed to achieve this reduction is $O(1) k d(\log y-\log x) / \log k$.

Proof. The successive calls can be done as follows. We first call subroutine2 $\left(k, J, n /\left(k x^{d}\right), y\right)$. By Lemma 4 it takes an expected number of $O(k)$ queries until the algorithm terminates. The sets $V_{j}, j \in J$, now have size at most $n /\left(k x^{d}\right)$. We next call subroutine2 $\left(k, J, n /\left(k^{2} x^{d}\right), y\right)$. After the $h$ th such call we are left with sets of size at most $n /\left(k^{h} x^{d}\right)$. For $h=d(\log y-$ 
$\log x) / \log k$ we have $k^{h} \geq(y / x)^{d}$. The total expected number of queries at this point is $O(1) k d(\log y-\log x) / \log k$.

Convention: In line 28 the pseudo-code of our main strategy, Algorithm 1, we write subroutine $2\left(x_{\ell-1}, J^{\prime}, n / x_{\ell}^{d}, x\right)$ if we appeal to subroutine2 repeatedly, until the size of each $V_{j}, j \in J^{\prime}$ has been reduced to $n / x_{\ell}^{d}$. Similarly in line 9 we write subroutine $2\left(x_{t}, J^{\prime}, 1, x\right)$ to appeal to subroutine 2 repeatedly until the size $V_{j}, j \in J^{\prime}$ has been reduced to one.

\section{B.4 Proof of Theorem 4}

It remains to show that the first phase of Algorithm 1 takes at most $O(n \log \log n)$ queries.

Theorem 6. Let $q \in n-\Theta(n / \log n)$. Using Algorithm 1, we can identify positions $\pi(1), \ldots, \pi(q)$ and the corresponding entries $z_{\pi(1)}, \ldots, z_{\pi(q)}$ of $z$ in these positions using at most $O(n \log \log n)$ queries.

We prove Theorem 6 . The proof of the required probabilistic statements is postponed to Sections B.5 and B.6.

If there is no failure in any call of Advance, the expected number of queries is bounded by

$$
\begin{aligned}
& \frac{q}{x_{t}}\left(\frac{x_{t} \log n}{\log x_{t}}+\frac{x_{t}}{x_{t-1}}\left(\frac{x_{t-1} d c\left(\log x_{t}-\log x_{t-1}\right)}{\log x_{t-1}}\right.\right. \\
& \left.\left.+\frac{x_{t-1}}{x_{t-2}}\left(\ldots+\frac{x_{2}}{x_{1}}\left(\frac{x_{1} d c\left(\log x_{2}-\log x_{1}\right)}{\log x_{1}}+x_{1} d \log x_{1}\right)\right)\right)\right) \\
\leq & n d c\left(\frac{\log n}{\log x_{t}}+\frac{\log x_{t}}{\log x_{t-1}}+\ldots+\frac{\log x_{2}}{\log x_{1}}+\log x_{1}-t\right),
\end{aligned}
$$

where $c$ is the constant hidden in the $O(1)$-term in Corollary 5 . To verify this formula, observe that each call of Advance $(i)$ makes $x_{i} / x_{i-1}$ calls to Advance $(i-1)$. After each such call we reduce the size of $x_{i-1}$ candidate sets from $n / x_{i-1}^{d}$ to $n / x_{i}^{d}$. By Corollary 5 , this requires at most $x_{i-1} d c\left(\log x_{i}-\log x_{i-1}\right) / \log x_{i-1}$ queries. The additional $x_{1} d \log x_{1}$ term accounts for the queries needed to move the sets from level 0 to level 1 ; i.e., it accounts for the queries caused by the calls Advance(1) through which we reduce the sizes of the $V_{i}$ s by the randomized binary search algorithm, subroutine 1 , to $n / x_{1}^{d}$-requiring $d \log x_{1}$ queries per call. Finally, the term $x_{t} \log n / \log x_{t}$ accounts for the final reduction of the $V_{i}$ s to a set containing only one single element (at this stage we shall finally have $V_{i}=\{\pi(i)\}$ ). More precisely, this term is $\left(x_{t}\left(\log n-d \log x_{t}\right)\right) / \log x_{t}$ but we settle for upper bounding this expression by the term given in the formula.

Next we need to bound the number of queries caused by failures. In Sections B.5 and B.6 we show that, on average, not too many failures happen. More precisely, we show that the expected number of level- $i$ failures is at most $n^{2} /\left((n-q)\left(x_{i}^{d-1}-1\right)\right)$. By Corollary 5 , each such level- $i$ failure causes an additional number of at most $1+x_{i} d c\left(\log x_{i+1}-\log x_{i}\right) / \log x_{i}$ queries; the 1 counts for the failured query in line 16; i.e., for the query through which we discover that $\pi\left(s^{*}+1\right) \in V_{1} \cup \ldots \cup V_{s^{*}}$. Thus, in total we get an additional number of at most

$$
\sum_{i=1}^{t} \frac{n^{2}}{(n-q)\left(x_{i}^{d-1}-1\right)}\left(1+\frac{x_{i} d c\left(\log x_{i+1}-\log x_{i}\right)}{\log x_{i}}\right)
$$

expected queries caused by failures.

We recall the settings of the $x_{i}$. We set $x_{1}:=\log n$ and we choose the $x_{j}$ such that $x_{j}=x_{j-1}^{2}$, $j=2, \ldots, t$. We further require that $\log x_{t}=\Omega(\log n)$, which can be achieved by choosing 
$t \in \Theta(\log \log n)$. In what follows, we do not specify the choice of $d$, but note that any choice $d \geq 4$ is good enough. With this parameter setting, formula (4) evaluates to

$$
n d c\left(\frac{\log n}{\log x_{t}}+2(t-1)+\log \log n-t\right)=O(n \log \log n)
$$

and, somewhat wasteful, we can bound formula (5) from above by

$$
\frac{n^{2} d c}{n-q} \sum_{i=1}^{t} x_{i}^{-(d-3)}=O(n \log n) \sum_{i=0}^{t-1} x_{1}^{-(d-3) 2^{i}}<O(n \log n)\left(x_{1}^{d-3}-1\right)^{-1}=O(n),
$$

where the first equation is by construction of the $x_{i} \mathrm{~s}$, the inequality uses the fact that the geometric sum is dominated by the first term, and the last equality stems from our choice $x_{1}=\log n$. This shows that the overall expected number of queries sums to $O(n \log \log n)+$ $O(n)=O(n \log \log n)$.

Key to the failure analyses in Sections B.5 and B.6 is the following observation.

Lemma 7. Each $V_{j}$ has the property that $V_{j} \backslash\{\pi(j)\}$ is random; i.e., is a random subset of $[n] \backslash\{\pi(j)\}$ of size $\left|V_{j}\right|-1$.

Proof. $V_{j}$ is initialized to [n]; thus the claim is true initially. In subroutine1, a random subset $F$ of $V_{j}$ is chosen and $V_{j}$ is reduced to $F$ (if $\pi(j) \in F$ ) or to $V_{j} \backslash F$ (if $\pi(j) \notin F$ ). In either case, the claim stays true. The same reasoning applies to subroutine2.

\section{B.5 Failures on Level One}

Lemma 8. A call of Advance(1) (i.e., lines 15 to 24) requires at most $x_{1}+x_{1} d \log x_{1}$ queries.

Proof. The two occasions where queries are made are in line 16 and in line 20. Line 16 is executed at most $x_{1}$ times, each time causing exactly one query. As shown in Lemma 11, each call to subroutine 1 in line 20 causes at most $d \log x_{1}$ queries. The subroutine is called at most $x_{1}$ times.

Lemma 9. Let $q \in n-\Theta(n / \log n)$ be the number of indices $i$ for which we determine $\pi(i)$ and $z_{\pi(i)}$ in the first phase.

The probability that any particular call of Advance(1) fails is at most $n(n-q)^{-1} \sum_{i=1}^{t} x_{i}^{-(d-1)}$.

Proof. A failure happens if we cannot increase the score of $x$ by flipping the bits in $[n] \backslash \cup_{i=1}^{s} V_{i}$; i.e., if $f(y) \leq s$ holds in line 16 of Algorithm 1. This is equivalent to $\pi(s+1) \in \cup_{i=1}^{s} V_{i}$.

Let $\ell$ be the number of indices $i \in[n]$ for which $V_{i}$ is on the last level; i.e., $\ell:=\mid\{i \in[n] \mid$ $\left.\left|V_{i}\right|=1\right\} \mid$ is the number of sets $V_{i}$ which have been reduced to singletons already. Note that these sets satisfy $V_{i}=\{\pi(i)\}$. Therefore, they cannot contain $\pi(s+1)$ and we do not need to take them into account.

By our random construction of the $V_{i}$ s (cf. Lemma 7), the probability that $\pi(s+1) \in \cup_{i=1}^{s} V_{i}$ is at most $\left|\cup_{i=\ell+1}^{s} V_{i}\right| /(n-\ell)$ and hence bounded by $\sum_{i=\ell+1}^{s}\left|V_{i}\right| /(n-\ell)$.

At any time during the run of Algorithm 1, there are at most $x_{i}$ sets $V_{j}$ on the $i$ th level. By construction, the size of each such level- $i$ set is at most $n x_{i}^{-d}$. Hence, we can bound the probability that $\pi(s+1) \in \cup_{i=1}^{s} V_{i}$ from above by

$$
\frac{n}{n-\ell} \sum_{i=1}^{t} x_{i}^{-(d-1)}
$$


By the exponential growth of the values $x_{1}, \ldots, x_{t}$ and the fact that we call line 16 a total number of $q<n$ times to improve upon the current best score, from Lemma 9 we immediately get the following.

Corollary 10. The expected number of level 1 failures is less than

$$
q n(n-q)^{-1}\left(x_{1}^{d-1}-1\right)^{-1} \leq n^{2}(n-q)^{-1}\left(x_{1}^{d-1}-1\right)^{-1} .
$$

\section{B.6 Failures at Higher Levels}

Lemma 11. As in Lemma 9 let $q$ be the number of indices $i$ for which we determine $\pi(i)$ and $z_{\pi(i)}$ in the first phase. Let $i \in[t]$.

The probability that a particular call of Advance( $i)$ fails (level $i$ failure) is at most $n(n-$ $q)^{-1}\left(x_{i}^{d-1}-1\right)^{-1}$.

Proof. This proof is similar to the one of Lemma 9: A failure on level $i$ occurs only if $\pi(s+1) \in$ $\cup_{j=1}^{s} V_{j}$ and the size of each candidate set $V_{1}, \ldots, V_{s}$ has been reduced already to at most $n / x_{i}^{d}$. There are at most $x_{j}$ candidate sets on each level $j \geq i$. By construction, the size of each candidate set on level $j$ is at most $n / x_{j}^{d}$. By Lemma 7, the probability that $\pi(s+1) \in \cup_{j=1}^{s} V_{j}$ is at most

$$
\frac{n}{n-\ell} \sum_{j=i}^{t} \frac{1}{x_{j}^{d-1}}
$$

where $\ell$ denotes again the number of sets that have been reduced to singletons already (i.e., the number of sets on level $t+1$ ).

By definition we have $x_{j} \geq x_{i}^{\left(2^{j-i}\right)}$ and in particular we have $x_{j} \geq x_{i}^{j-i}$. Therefore expression (6) can be bounded from above by

$$
\frac{n}{n-\ell} \sum_{j=1}^{t-i}\left(\frac{1}{x_{i}^{d-1}}\right)^{j}<\frac{n}{n-\ell}\left(x_{i}^{d-1}-1\right)^{-1} .
$$

By the same reasoning as in Section B.5, from Lemma 11 we immediately get the following.

Corollary 12. Let $i \in[t]$.

The expected number of level $i$ failures is less than

$$
n q(n-q)^{-1}\left(x_{i}^{d-1}-1\right)^{-1} \leq n^{2}(n-q)^{-1}\left(x_{i}^{d-1}-1\right)^{-1} .
$$

\section{Details on the Lower Bound, Section 5}

\section{C.1 Candidate Sets in the Lower Bound Context}

At node $v$ of the decision tree, the candidate set $V_{i}^{v}$, intuitively corresponds to the possible values of $\pi(i)$. At the root node $r$, we have $V_{i}^{r}=[n]$ for all $i$. Let $v$ be a node in the tree and let $w_{0}, \ldots, w_{n}$ be its children $\left(w_{i}\right.$ is traversed when the score $i$ is returned). Let $P_{0}^{v}$ (resp. $\left.P_{1}^{v}\right)$ 
be the set of positions in $x_{v}$ that contain 0 (resp. 1). Thus, formally, $P_{0}^{v}=\left\{i \mid x_{v}[i]=0\right\}$ and $P_{1}^{v}=\left\{i \mid x_{v}[i]=1\right\} .^{2}$ The precise definition of candidate sets is as follows:

$$
V_{i}^{w_{j}}= \begin{cases}V_{i}^{v} \cap P_{i \bmod 2}^{v} & \text { if } i \leq j \\ V_{i}^{v} \cap P_{j \bmod 2}^{v} & \text { if } i=j+1 \\ V_{i}^{v} & \text { if } i>j+1 .\end{cases}
$$

Note that, as was the case with the candidate sets defined in Section 2, not all values in a candidate set could be valid. But as with the upper bound case, the candidate sets have some very useful properties. These properties are also slightly different from the ones observed before, due to the fact that some extra information has been announced to the query algorithm. We reiterate the definition of an active candidate set. We say that a candidate set $V_{i}^{v}$ is active (at $v$ ) if the following conditions are met: (i) at some ancestor node $u$ of $v$, we have $F\left(x_{u}\right)=i-1$ and (ii) at every ancestor node $w$ of $u$, we have $F\left(x_{w}\right)<i-1$, and (iii) $i<\min \left\{n / 3, \max _{v}\right\}$. We call $V_{\max _{v}+1}^{v}$ pseudo-active (at $v$ ). The following theorem can be proved using similar ideas as in Theorem 1

Lemma 8. The candidate sets have the following properties:

(i) Two candidate sets $V_{i}^{v}$ and $V_{j}^{v}$ with $i<j \leq \max _{v}$ and $i \not \equiv j$ are disjoint.

(ii) An active candidate set $V_{j}^{v}$ is disjoint from any candidate set $V_{i}$ provided $i<j<\max _{v}$.

(iii) The candidate set $V_{i}^{v}, i \leq \max _{v}$ is contained in the set $V_{\max _{v}+1}^{v}$ if $i \equiv \max _{v}$ and is disjoint from it if $i \not \equiv \max _{v}$.

(iv) For two candidate sets $V_{i}^{v}$ and $V_{j}^{v}, i<j$, if $V_{i}^{v} \cap V_{j}^{v} \neq \emptyset$ then $V_{i}^{v} \subset V_{j}^{v}$.

Proof. Let $w$ be the ancestor of $v$ where the function returns score $\max _{v}$.

To prove (i), observe that in $w$, one of $V_{i}^{w}$ and $V_{j}^{w}$ is intersected by $P_{0}^{w}$ while the other is intersected by $P_{1}^{w}$ and thus they are made disjoint.

To prove (ii), we can assume $i \equiv j$ as otherwise the result follows from the previous case. Let $u$ be the ancestor of $v$ such that $F\left(x_{u}\right)=j-1$ and that in any ancestor of $u$, the score returned by the function is smaller than $j-1$. At $u, V_{j}^{u}$ is intersected with $P_{j-1 \bmod 2}^{u}$ while $V_{i}^{v}$ is intersected with $P_{i \bmod 2}^{u}$. Since $i \equiv j$, it follows that they are again disjoint.

For (iii), the latter part follows as in (i). Consider an ancestor $v^{\prime}$ of $v$ and let $w_{j}$ be the $j$ th child of $v^{\prime}$ that is also an ancestor of $v$. We use induction and we assume $V_{i}^{v^{\prime}} \subset V_{\max _{v}+1}^{v^{\prime}}$. If $j<\max _{v}$, then $V_{\max _{v}+1}^{v^{\prime}}=V_{\max _{v}+1}^{w_{j}}$ which means $V_{i}^{w_{j}} \subset V_{\max _{v}+1}^{w_{j}}$. If $j=\max _{v}$, then $V_{\max _{v}+1}^{w_{j}}=V_{\max _{v}+1}^{v^{\prime}} \cap P_{\max _{v}}^{v^{\prime}} \bmod 2$ and notice that in this case also $V_{i}^{w_{j}}=V_{i}^{v^{\prime}} \cap P_{i \bmod 2}^{v^{\prime}}$ which still implies $V_{i}^{w_{j}} \subset V_{\max _{v}+1}^{w_{j}}$.

To prove (iv), first observe that the statement is trivial if $i \not \equiv j$. Also, if the function returns score $j-1$ at any ancestor of $v$, then by the same argument used in (ii) it is possible to show that $V_{i}^{v} \cap V_{j}^{v}=\emptyset$. Thus assume $i \equiv j$ and the function never returns value $j-1$. In this case, it is easy to see that an inductive argument similar to (iii) proves that $V_{i}^{v^{\prime}} \subset V_{j}^{v^{\prime}}$ for every ancestor $v^{\prime}$ of $v$.

Corollary 13. Every two distinct active candidate sets $V_{i}^{v}$ and $V_{j}^{v}$ are disjoint.

Lemma 14. Consider a candidate set $V_{i}^{v}$ and let $i_{1}<\cdots<i_{k}<i$ be the indices of candidate sets that are subsets of $V_{i}^{v}$. Let $\sigma:=\left(\sigma_{1}, \cdots, \sigma_{i}\right)$ be a sequence without repetition from $[n]$ and let $\sigma^{\prime}:=\left(\sigma_{1}, \cdots, \sigma_{i-1}\right)$. Let $n_{\sigma}$ and $n_{\sigma^{\prime}}$ be the number of permutations in $S_{v}$ that have $\sigma$ and $\sigma^{\prime}$ as a prefix, respectively. If $n_{\sigma}>0$, then $n_{\sigma^{\prime}}=\left(\left|V_{i}^{v}\right|-k\right) n_{\sigma}$.

\footnotetext{
${ }^{2}$ To prevent our notations from becoming too overloaded, here and in the remainder of the section we write $x=(x[1], \ldots, x[n])$ instead of $x=\left(x_{1}, \ldots, x_{n}\right)$
} 
Proof. Consider a permutation $\pi \in S_{v}$ that has $\sigma$ as a prefix. This implies $\pi(i) \in V_{i}^{v}$. For an element $s \in V_{i}^{v}, s \neq i_{j}, 1 \leq j \leq k$, let $\pi_{s}$ be the permutation obtained from $\pi$ by placing $s$ at position $i$ and placing $\pi(i)$ where $s$ used to be. Since $s \neq i_{j}, 1 \leq j \leq k$, it follows that $\pi_{s}$ has $\sigma^{\prime}$ as prefix and since $s \in V_{i}^{v}$ it follows that $\pi_{s} \in S_{v}$. It is easy to see that for every permutation in $S_{v}$ that has $\sigma$ as a prefix we will create $\left|V_{i}^{v}\right|-k$ different permutations that have $\sigma^{\prime}$ as a prefix and all these permutations will be distinct. Thus, $n_{\sigma^{\prime}}=\left(\left|V_{i}^{v}\right|-k\right) n_{\sigma}$.

Corollary 15. Consider a candidate set $V_{i}^{v}$ and let $i_{1}<\cdots<i_{k}<i$ be the indices of candidate sets that are subsets of $V_{i}^{v}$. Let $\sigma^{\prime}:=\left(\sigma_{1}, \cdots, \sigma_{i-1}\right)$ be a sequence without repetition from $[n]$ and let $\sigma_{1}:=\left(\sigma_{1}, \cdots, \sigma_{i-1}, s_{1}\right)$ and $\sigma_{2}:=\left(\sigma_{1}, \cdots, \sigma_{i-1}, s_{2}\right)$ in which $s_{1}, s_{2} \in V_{i}^{v}$. Let $n_{\sigma_{1}}$ and $n_{\sigma_{2}}$ be the number of permutations in $S_{v}$ that have $\sigma_{1}$ and $\sigma_{2}$ as a prefix, respectively. If $n_{\sigma_{1}}, n_{\sigma_{2}}>0$, then $n_{\sigma_{1}}=n_{\sigma_{2}}$.

Proof. Consider a sequence $s_{1}, \cdots, s_{i}$ without repetition from $[n]$ such that $s_{j} \in V_{j}^{v}, 1 \leq j \leq i$. By the previous lemma $\operatorname{Pr}\left[\Pi(1)=s_{1} \wedge \cdots \wedge \Pi(i-1)=s_{i-1} \wedge \Pi(i)=s_{i}\right]=\operatorname{Pr}[\Pi(1)=$ $\left.s_{1} \wedge \cdots \wedge \Pi(i-1)=s_{i-1}\right] \cdot\left(1 /\left|V_{i}^{v}\right|\right)$.

Corollary 16. If $V_{i}^{v}$ is active, then we have:

(i) $\Pi(i)$ is independent of $\Pi(1), \cdots, \Pi(i-1)$.

(ii) $\Pi(i)$ is uniformly distributed in $V_{i}^{v}$.

\section{C.2 Potential Function Analysis}

The main result of this section is the following.

Lemma 9. Let $v$ be a node in $T$ and let $\boldsymbol{i}_{v}$ be the random variable giving the value of $F\left(x_{v}\right)$ when $\Pi \in S_{v}$ and 0 otherwise. Also let $w_{0}, \ldots, w_{n}$ denote the children of $v$, where $w_{j}$ is the child reached when $F\left(x_{v}\right)=j$. Then, $\mathbb{E}\left[\varphi\left(w_{\boldsymbol{i}_{v}}\right)-\varphi(v) \mid \Pi \in S_{v}\right]=O(1)$.

We write

$$
\mathbb{E}\left[\varphi\left(w_{\mathbf{i}_{v}}\right)-\varphi(v) \mid \Pi \in S_{v}\right]=\sum_{a=0}^{n} \operatorname{Pr}\left[F\left(x_{v}\right)=a \mid \Pi \in S_{v}\right]\left(\varphi\left(w_{a}\right)-\varphi(v)\right) .
$$

Before presenting the formal analysis of this potential function, we note that we have two main cases: $F\left(x_{v}\right) \leq \max _{v}$ and $F\left(x_{v}\right)>\max _{v}$. In the first case, the maximum score will not increase in $w_{a}$ which means $w_{a}$ will have the same set of active candidate sets. In the second case, the pseudo-active candidate set $V_{\max _{v}+1}^{v}$ will turn into an active set $V_{\max _{v}+1}^{w_{a}}$ at $w_{a}$ and $w_{a}$ will have a new pseudo-active set. While this second case looks more complicated, it is in fact the less interesting part of the analysis since the probability of suddenly increasing the score by $\alpha$ is extremely small (we will show that it is roughly $O\left(2^{-\Omega(\alpha)}\right)$ ) which subsumes any significant potential increase for values of $a>\max _{v}$. As discussed, we divide the above summation into two parts: one for $a \leq \max _{v}$ and another for $a>\max _{v}$ :

$$
\begin{array}{r}
\mathbb{E}\left[\varphi\left(w_{\mathbf{i}_{v}}\right)-\varphi(v) \mid \Pi \in S_{v}\right]= \\
\sum_{a=0}^{\max _{v}} \operatorname{Pr}\left[F\left(x_{v}\right)=a \mid \Pi \in S_{v}\right]\left(\varphi\left(w_{a}\right)-\varphi(v)\right)+ \\
\sum_{a=1}^{n / 3-\max _{v}} \operatorname{Pr}\left[F\left(x_{v}\right)=\max _{v}+a \mid \Pi \in S_{v}\right]\left(\varphi\left(w_{a}\right)-\varphi(v)\right) .
\end{array}
$$

To bound the above two summations, it is clear that we need to handle $\operatorname{Pr}\left[F\left(x_{v}\right)=a \mid \Pi \in S_{v}\right]$. In the next section, we will prove lemmas that will do this. 


\section{C.2.1 Bounding Probabilities}

Let $a_{1}, \ldots, a_{\left|A_{v}\right|}$ be the indices of active candidate sets at $v$ sorted in increasing order. We also define $a_{\left|A_{v}\right|+1}=\max _{v}+1$. For a candidate set $V_{i}^{v}$, and a Boolean $b \in\{0,1\}$, let $V_{i}^{v}(b)=$ $\left\{j \in V_{i}^{v} \mid x_{v}[j]=b\right\}$. Clearly, $\left|V_{i}^{v}(0)\right|+\left|V_{i}^{v}(1)\right|=\left|V_{i}^{v}\right|$. For even $a_{i}, 1 \leq i \leq\left|A_{v}\right|$, let $\varepsilon_{i}=$ $\left|V_{i}^{v}(1)\right| /\left|V_{i}^{v}\right|$ and for odd $i$ let $\varepsilon_{i}=\left|V_{i}^{v}(0)\right| /\left|V_{i}^{v}\right|$. This definition might seem strange but is inspired by the following observation.

Lemma 17. For $i \leq\left|A_{v}\right|, \operatorname{Pr}\left[F\left(x_{v}\right)=a_{i}-1 \mid \Pi \in S_{v} \wedge F\left(x_{v}\right)>a_{i}-2\right]=\varepsilon_{i}$.

Proof. Note that $F\left(x_{v}\right)=a_{i}-1$ happens if and only if $F\left(x_{v}\right)>a_{i}-2$ and $x_{v}\left[\Pi\left(a_{i}\right)\right] \not \equiv a_{i}$. Since $V_{a_{i}}^{v}$ is an active candidate set, the lemma follows from Corollary 16 and the definition of $\varepsilon_{i}$.

Let $\varepsilon_{i}^{\prime}:=\operatorname{Pr}\left[a_{i} \leq F\left(x_{v}\right)<a_{i+1}-1 \mid \Pi \in S_{v} \wedge F\left(x_{v}\right) \geq a_{i}\right]$. Note that $\varepsilon_{i}^{\prime}=0$ if $a_{i+1}=a_{i}+1$.

Lemma 18. For $i \leq\left|A_{v}\right|$ we have $\left|V_{a_{i}}^{w_{j}}\right|=\left|V_{a_{i}}^{v}\right|$ for $j<a_{i}-1,\left|V_{a_{i}}^{w_{j}}\right|=\varepsilon_{i}\left|V_{a_{i}}^{v}\right|$ for $j=a_{i}-1$, and $\left|V_{a_{i}}^{w_{j}}\right|=\left(1-\varepsilon_{i}\right)\left|V_{a_{i}}^{v}\right|$ for $j>a_{i}-1$. Also,

$$
\begin{aligned}
\operatorname{Pr}\left[F\left(x_{v}\right)=a_{i}-1 \mid \Pi \in S_{v}\right] & =\varepsilon_{i} \Pi_{j=1}^{i-1}\left(1-\varepsilon_{j}\right)\left(1-\varepsilon_{j}^{\prime}\right), \\
\operatorname{Pr}\left[a_{i} \leq F\left(x_{v}\right)<a_{i+1}-1 \mid \Pi \in S_{v}\right] & =\varepsilon_{i}^{\prime}\left(1-\varepsilon_{i}\right) \Pi_{j=1}^{i-1}\left(1-\varepsilon_{j}\right)\left(1-\varepsilon_{j}^{\prime}\right),
\end{aligned}
$$

Proof. Using Lemma 17, it is verified that

$$
\begin{aligned}
\operatorname{Pr}\left[F\left(x_{v}\right)>a_{i}-1 \mid \Pi \in S_{v}\right] & =\operatorname{Pr}\left[F\left(x_{v}\right)>a_{i}-2 \wedge F\left(x_{v}\right) \neq a_{i}-1 \mid \Pi \in S_{v}\right] \\
& =\operatorname{Pr}\left[F\left(x_{v}\right) \neq a_{i}-1 \mid F\left(x_{v}\right)>a_{i}-2 \wedge \Pi \in S_{v}\right] . \\
\operatorname{Pr}\left[F\left(x_{v}\right)>a_{i}-2 \mid \Pi \in S_{v}\right] & =\left(1-\varepsilon_{i}\right) \operatorname{Pr}\left[F\left(x_{v}\right)>a_{i}-2 \mid \Pi \in S_{v}\right] .
\end{aligned}
$$

Similarly, using the definition of $\varepsilon_{i}^{\prime}$ we can see that

$$
\begin{aligned}
& \operatorname{Pr}\left[F\left(x_{v}\right)>a_{i}-2 \mid \Pi \in S_{v}\right] \\
& =\operatorname{Pr}\left[F\left(x_{v}\right) \notin\left\{a_{i-1}, \ldots, a_{i}-2\right\} \wedge F\left(x_{v}\right)>a_{i-1}-1 \mid \Pi \in S_{v}\right] \\
& =\operatorname{Pr}\left[F\left(x_{v}\right) \notin\left\{a_{i-1}, \ldots, a_{i}-2\right\} \mid F\left(x_{v}\right)>a_{i-1}-1 \wedge \Pi \in S_{v}\right] \operatorname{Pr}\left[F\left(x_{v}\right)>a_{i-1}-1 \mid \Pi \in S_{v}\right] \\
& =\left(1-\varepsilon_{i-1}^{\prime}\right) \operatorname{Pr}\left[F\left(x_{v}\right)>a_{i-1}-1 \mid \Pi \in S_{v}\right] .
\end{aligned}
$$

Using these, we get that

$$
\operatorname{Pr}\left[F\left(x_{v}\right)>a_{i}-1 \mid \Pi \in S_{v}\right]=\left(1-\varepsilon_{i}\right) \Pi_{j=1}^{i-1}\left(1-\varepsilon_{j}\right)\left(1-\varepsilon_{j}^{\prime}\right)
$$

and

$$
\operatorname{Pr}\left[F\left(x_{v}\right)>a_{i}-2 \mid \Pi \in S_{v}\right]=\Pi_{j=1}^{i-1}\left(1-\varepsilon_{j}\right)\left(1-\varepsilon_{j}^{\prime}\right) .
$$

Equalities (9) and (10) follow from combining these bounds with Lemma 17. The rest of the lemma follows directly from the definition of $\varepsilon_{i}$ and the candidate sets.

Lemma 19. Let $b \in\{0,1\}$ be such that $b \equiv \max _{v}$ and let $k:=\left|V_{\max _{v}+1}^{v}(b)\right|$. Then,

$$
\operatorname{Pr}\left[F\left(x_{v}\right)=\max _{v} \mid \Pi \in S_{v}\right]=\frac{k-\operatorname{Con}_{v}}{\left|V_{\max _{v}+1}^{v}\right|-\operatorname{Con}_{v}} \prod_{i=1}^{\left|A_{v}\right|}\left(1-\varepsilon_{i}\right)\left(1-\varepsilon_{i}^{\prime}\right) .
$$

Proof. Conditioned on $F\left(x_{v}\right)>\max _{v}-1, F\left(x_{v}\right)$ will be equal to $\max _{v}$ if $x_{v}\left[\Pi\left(\max _{v}+1\right)\right]=b$. By definition, the number of positions in $V_{\max _{v}+1}^{v}$ that satisfy this is $k$. However, $V_{\max _{v}+1}^{v}$ contains $\mathrm{Con}_{v}$ candidate sets but since $V_{\max _{v}+1}^{v}$ can only contain a candidate set $V_{i}^{v}$ if $i \equiv \max _{v}$ (by Lemma 8), it follows from Lemma 14 that $\operatorname{Pr}\left[F\left(x_{v}\right)=\max _{v} \mid \Pi \in S_{v} \wedge F\left(x_{v}\right)>\max _{v}-1\right]=$ $\left(k-\mathrm{Con}_{v}\right) /\left(\left|V_{\max _{v}+1}^{v}\right|-\mathrm{Con}_{v}\right)$. The lemma then follows from the previous lemma. 
Lemma 20. Let $b \in\{0,1\}$ be such that $b \equiv \max _{v}$ and let $k:=\left|V_{\max _{v}+1}^{v}(b)\right|$. Then,

$$
\operatorname{Pr}\left[F\left(x_{v}\right)>\max _{v} \mid \Pi \in S_{v}\right] \leq \frac{\left|V_{\max _{v}+1}^{v}\right|-k}{\left|V_{\max _{v}+1}^{v}\right|-\mathrm{Con}_{v}} .
$$

Proof. From the previous lemma we have that $\operatorname{Pr}\left[F\left(x_{v}\right)=\max _{v} \mid \Pi \in S_{v} \wedge F\left(x_{v}\right)>\max _{v}-1\right]=$ $\left(k-\mathrm{Con}_{v}\right) /\left(\left|V_{\max _{v}+1}^{v}\right|-\mathrm{Con}_{v}\right)$. Thus,

$$
\operatorname{Pr}\left[F\left(x_{v}\right)>\max _{v} \mid \Pi \in S_{v} \wedge F\left(x_{v}\right)>\max _{v}-1\right]=\frac{\left|V_{\max _{v}+1}^{v}\right|-k}{\left|V_{\max _{v}+1}^{v}\right|-\mathrm{Con}_{v}} .
$$

Using induction, it is possible to prove that

$$
\operatorname{Pr}\left[F\left(x_{v}\right)>\max _{v} \mid \Pi \in S_{v}\right] \frac{\left|V_{\max _{v}+1}^{v}\right|-k}{\left|V_{\max _{v}+1}^{v}\right|-\operatorname{Con}_{v}} \prod_{i=1}^{\left|A_{v}\right|}\left(1-\varepsilon_{i}\right)\left(1-\varepsilon_{i}^{\prime}\right),
$$

which implies the lemma.

Remember that $P_{0}^{v}$ (resp. $P_{1}^{v}$ ) are the set of positions in $x_{v}$ that contain 0 (resp. 1).

Lemma 21. Let $x_{0}=\left|P_{0}^{v}\right|$ and $x_{1}=\left|P_{1}^{v}\right|$. Let $b_{i}$ be a Boolean such that $b_{i} \equiv i$. For $a \geq \max _{v}+2$

$$
\operatorname{Pr}\left[F\left(x_{v}\right)=a \mid \Pi \in S_{v}\right] \leq\left(\prod_{i=\max _{v}+2}^{a} \frac{x_{b_{i}}-\lfloor i / 2\rfloor}{n-i+1}\right)\left(1-\frac{x_{b_{a+1}}-\lfloor(a+1) / 2\rfloor}{n-a}\right) .
$$

Proof. Notice that we have $V_{i}^{v}=[n]$ for $i \geq \max _{v}+2$ which means $i-1$ is the number of candidates sets $V_{j}^{v}$ contained in $V_{i}^{v}$ and among those $\lfloor i / 2\rfloor$ are such that $i \equiv j$. Consider a particular prefix $\sigma=\left(\sigma_{1}, \cdots, \sigma_{i-1}\right)$ such that there exists a permutation $\pi \in S_{v}$ that has $\sigma$ as a prefix. This implies that $\sigma_{j} \in P_{b_{j}}^{v}$. Thus, it follows that there are $x_{b_{i}}-\lfloor i / 2\rfloor$ elements $s \in P_{b_{i}}^{v}$ such that the sequences $\left(\sigma_{1}, \cdots, \sigma_{i-1}, s\right)$ can be the prefix of a permutation in $S_{v}$. Thus by Corollary 15, and for $i \geq \max _{v}+2$,

$$
\operatorname{Pr}\left[F\left(x_{v}\right)=i-1 \mid \Pi \in S_{v} \wedge F\left(x_{v}\right) \geq i-1\right]=1-\frac{x_{b_{i}}-\lfloor i / 2\rfloor}{n-i+1}
$$

and

$$
\operatorname{Pr}\left[F\left(x_{v}\right) \geq i \mid \Pi \in S_{v} \wedge F\left(x_{v}\right) \geq i-1\right]=\frac{x_{b_{i}}-\lfloor i / 2\rfloor}{n-i+1} .
$$

Corollary 22. For $\max _{v}+1 \leq a \leq n / 3$ we have

$$
\operatorname{Pr}\left[F\left(x_{v}\right)=a \mid \Pi \in S_{v}\right]=2^{-\Omega\left(a-\max _{v}\right)} \cdot\left(1-\frac{x_{b_{a+1}}-\lfloor(a+1) / 2\rfloor}{n-a}\right) .
$$

Proof. Since $x_{b_{i}}+x_{b_{i+1}}=n$, it follows that

$$
\left(\frac{x_{b_{i}}-\lfloor i / 2\rfloor}{n-i+1}\right)\left(\frac{x_{b_{i+1}}-\lfloor i / 2\rfloor}{n-i+2}\right) \leq\left(\frac{x_{b_{i}}-\lfloor i / 2\rfloor}{n-i+1}\right)\left(\frac{x_{b_{i+1}}-\lfloor i / 2\rfloor}{n-i+1}\right) \leq \frac{1}{2} .
$$

Now we analyze the potential function. 


\section{C.2.2 Bounding (7)}

We have,

$$
\varphi\left(w_{a}\right)-\varphi(v)=\log \frac{\log \frac{2 n}{\left|V_{\max _{w_{a}}+1}^{w_{a}}\right|-\operatorname{Con}_{w_{a}}}}{\log \frac{2 n}{\left|V_{\max _{v}+1}^{v}\right|-\operatorname{Con}_{v}}}+\sum_{j \in A_{v}} \log \frac{\log \frac{2 n}{\left|V_{j}^{w_{a}}\right|}}{\log \frac{2 n}{\left|V_{j}^{v}\right|}} .
$$

When $a \leq \max _{v}$ we have, $\max _{v}=\max _{w_{a}}$ and $\operatorname{Con}_{v}=\operatorname{Con}_{w_{a}}$. For $a<\max _{v}$, we also have $V_{\max _{v}+1}^{w_{a}}=V_{\max _{v}+1}^{v}$. It is clear from (11) that for $a_{i} \leq a<a_{i+1}-1$, all the values of $\varphi\left(w_{a}\right)-\varphi(v)$ will be equal. Thus,

$$
\begin{aligned}
(7)= & \sum_{i=1}^{\left|A_{v}\right|} \operatorname{Pr}\left[F\left(x_{v}\right)=a_{i}-1 \mid \Pi \in S_{v}\right]\left(\varphi\left(w_{a_{i}-1}\right)-\varphi(v)\right)+ \\
& \sum_{i=1}^{\left|A_{v}\right|} \operatorname{Pr}\left[a_{i} \leq F\left(x_{v}\right)<a_{i+1}-1 \mid \Pi \in S_{v}\right]\left(\varphi\left(w_{a_{i}}\right)-\varphi(v)\right)+ \\
& \operatorname{Pr}\left[F\left(x_{v}\right)=\max _{v} \mid \Pi \in S_{v}\right]\left(\varphi\left(w_{\max _{v}}\right)-\varphi(v)\right) .
\end{aligned}
$$

Analyzing (12) We write (12) using (11) and Lemma 18(9). Using inequalities, $1-x \leq e^{-x}$, for $0 \leq x \leq 1, \log (1+x) \leq x$ for $x \geq 0$, and $\sum_{1 \leq i \leq k} y_{i} \log 1 / y_{i} \leq Y \log (k / Y)$ for $y_{i} \geq 0$ and $Y=\sum_{1 \leq i \leq k} y_{i}$, we get the following:

$$
\begin{aligned}
& (12)=\sum_{i=1}^{\left|A_{v}\right|} \varepsilon_{i} \prod_{j=1}^{i-1}\left(1-\varepsilon_{j}\right)\left(1-\varepsilon_{j}^{\prime}\right)\left(\sum_{j=1}^{i} \log \frac{\log \frac{2 n}{\left|V_{a_{j}}^{w_{a}-1}\right|}}{\log \frac{2 n}{\left|V_{a_{j}}^{v}\right|}}\right)= \\
& \sum_{i=1}^{\left|A_{v}\right|} \varepsilon_{i} \prod_{j=1}^{i-1}\left(1-\varepsilon_{j}\right)\left(1-\varepsilon_{j}^{\prime}\right)\left(\log \frac{\log \frac{2 n}{\left|V_{a_{i}}^{w_{a_{i}}-1}\right|}}{\log \frac{2 n}{\left|V_{a_{i}}^{v}\right|}}+\sum_{j=1}^{i-1} \log \frac{\log \frac{2 n}{\left|V_{a_{j}}^{w_{a_{i}-1}}\right|}}{\log \frac{2 n}{\left|V_{a_{j}}^{v}\right|}}\right)= \\
& \sum_{i=1}^{\left|A_{v}\right|} \varepsilon_{i} \prod_{j=1}^{i-1}\left(1-\varepsilon_{j}\right)\left(1-\varepsilon_{j}^{\prime}\right)\left(\log \frac{\log \frac{2 n}{\left|V_{a_{i}}^{v}\right|}+\log \frac{1}{\varepsilon_{i}}}{\log \frac{2 n}{\left|V_{a_{i}}^{v}\right|}}+\sum_{j=1}^{i-1} \log \frac{\log \frac{2 n}{\left|V_{a_{j}}^{v}\right|}+\log \frac{1}{1-\varepsilon_{j}}}{\log \frac{2 n}{\left|V_{a_{j}}^{v}\right|}}\right)= \\
& \sum_{i=1}^{\left|A_{v}\right|} \varepsilon_{i} \prod_{j=1}^{i-1}\left(1-\varepsilon_{j}\right)\left(1-\varepsilon_{j}^{\prime}\right)\left(\log \left(1+\frac{\log \frac{1}{\varepsilon_{i}}}{\log \frac{2 n}{\left|V_{a_{i}}^{v}\right|}}\right)+\sum_{j=1}^{i-1} \log \left(1+\frac{\log \frac{1}{1-\varepsilon_{j}}}{\log \frac{2 n}{\left|V_{a_{j}}^{v}\right|}}\right)\right) \leq \\
& \sum_{i=1}^{\left|A_{v}\right|} \varepsilon_{i} \prod_{j=1}^{i-1}\left(1-\varepsilon_{j}\right)\left(\log \left(1+\frac{\log \frac{1}{\varepsilon_{i}}}{\log \frac{2 n}{\left|V_{a_{i}}^{v}\right|}}\right)+\sum_{j=1}^{i-1} \log \left(1+\log \frac{1}{1-\varepsilon_{j}}\right)\right) \leq \\
& \sum_{i=1}^{\left|A_{v}\right|} \varepsilon_{i} \prod_{j=1}^{i-1}\left(1-\varepsilon_{j}\right)\left(\log \left(1+\frac{\log \frac{1}{\varepsilon_{i}}}{\log \frac{2 n}{\left|V_{a_{i}}^{v}\right|}}\right)+\sum_{j=1}^{i-1} \log \frac{1}{1-\varepsilon_{j}}\right)= \\
& \sum_{i=1}^{\left|A_{v}\right|} \varepsilon_{i} \log \left(1+\frac{\log \frac{1}{\varepsilon_{i}}}{\log \frac{2 n}{\left|V_{a_{i}}^{v}\right|}}\right) \prod_{j=1}^{i-1}\left(1-\varepsilon_{j}\right)+ \\
& \sum_{i=1}^{\left|A_{v}\right|} \varepsilon_{i} \prod_{j=1}^{i-1}\left(1-\varepsilon_{j}\right)\left(\sum_{j=1}^{i-1} \log \frac{1}{1-\varepsilon_{j}}\right) .
\end{aligned}
$$


To bound (15), we use the fact that any two active candidate sets are disjoint. We break the summation into smaller chunks. Observe that $\prod_{j=1}^{i-1}\left(1-\varepsilon_{j}\right) \leq e^{-\sum_{j=1}^{i-1} \varepsilon_{j}}$. Thus, let $J_{t}, t \geq 0$, be the set of indices such that for each $i \in J_{t}$ we have $2^{t}-1 \leq \sum_{j=1}^{i-1} \varepsilon_{j}<2^{t+1}$. Now define $J_{t, k}=\left\{i \in J_{t}\left|n / 2^{k+1} \leq\right| V_{a_{i}}^{v} \mid \leq n / 2^{k}\right\}$, for $0 \leq k \leq \log n$ and let $s_{t, k}=\sum_{i \in J_{t, k}} \varepsilon_{i}$. Observe that by the disjointness of two active candidate sets, $\left|J_{t, k}\right| \leq 2^{k+1}$.

$$
\begin{array}{r}
(15)=\sum_{t=0}^{\log n \log n} \sum_{k=1} \sum_{i \in J_{t, k}} \varepsilon_{i} \log \left(1+\frac{\log \frac{1}{\varepsilon_{i}}}{\log \frac{2 n}{\left|V_{a_{i}}\right|}}\right) \prod_{j=1}^{i-1}\left(1-\varepsilon_{j}\right) \leq \\
\sum_{t=0}^{n} \sum_{k=1}^{\log n} \sum_{i \in J_{t, k}} \varepsilon_{i} \log \left(1+\frac{\log \frac{1}{\varepsilon_{i}}}{k}\right) e^{-\sum_{j=1}^{i-1} \varepsilon_{j}} \leq \\
\sum_{t=0}^{n} \sum_{k=1}^{\log n} \sum_{i \in J_{t, k}} \varepsilon_{i} \frac{\log \frac{1}{\varepsilon_{i}}}{k} e^{-2^{t}+1} \leq \sum_{t=0}^{n} \sum_{k=1}^{\log n} \frac{s_{t, k} \log \frac{\left|J_{t, k}\right|}{s_{t, k}}}{k} e^{-2^{t}+1} \leq \\
\sum_{t=0}^{n} \sum_{k=1}^{\log n} \frac{s_{t, k}(k+1)+s_{t, k} \log \frac{1}{s_{t, k}}}{k} e^{-2^{t}+1} \leq \sum_{t=0}^{n} 2^{t+2} e^{-2^{t}+1}+\sum_{t=0}^{n} \sum_{k=1}^{\log n} \frac{s_{t, k} \log \frac{1}{s_{t, k}}}{k} e^{-2^{t}+1} \leq \\
O(1)+\sum_{t=0}^{n} \sum_{r=1}^{\log \log n} \sum_{k=2^{r-1}}^{2^{r}} \frac{s_{t, k} \log \frac{1}{s_{t, k}}}{2^{r-1}} e^{-2^{t}+1} .
\end{array}
$$

Now define $S_{t, r}=\sum_{2^{r-1} \leq k<2^{r}} s_{t, k}$. Remember that $\sum_{r=1}^{\log \log n} S_{t, r}<2^{t+1}$. Thus,

$$
\begin{array}{r}
(15) \leq O(1)+\sum_{t=0}^{n} \sum_{r=1}^{\log \log n} \frac{S_{t, r} \log \frac{2^{r-1}}{S_{t, r}}}{2^{r-1}} e^{-2^{t}+1}= \\
O(1)+\sum_{t=0}^{n} \sum_{r=1}^{\log \log n} \frac{S_{t, r}(r-1)+S_{t, r} \log \frac{1}{S_{t, r}}}{2^{-2^{t}+1}} \leq \\
O(1)+\sum_{t=0}^{n} \sum_{r=1}^{\log \log n} \frac{2^{t+1}(r-1)}{2^{r-1}} e^{-2^{t}+1}+\sum_{t=0}^{n} \sum_{r=1}^{\log \log n} \frac{1}{2^{r-1}} e^{-2^{t}+1}=O(1) .
\end{array}
$$

To bound (16), define $J_{t}$ as before and let $p_{i}=\prod_{j=1}^{i-1} 1 /\left(1-\varepsilon_{j}\right)$. Observe that the function $\log (1 /(1-x))$ is a convex function which means if $s_{i}:=\sum_{j=1}^{i-1} \varepsilon_{j}$ is fixed, then $\prod_{j=1}^{i-1} 1 /\left(1-\varepsilon_{j}\right)$ is minimized when $\varepsilon_{j}=s_{i} /(i-1)$. Thus,

$$
p_{i} \geq\left(\frac{1}{1-\frac{s_{i}}{i-1}}\right)^{i-1} \geq\left(1+\frac{s_{i}}{i-1}\right)^{i-1} \geq 1+\left(\begin{array}{c}
i-1 \\
j
\end{array}\right)\left(\frac{s_{i}}{i-1}\right)^{j}
$$

in which $j$ can be chosen to be any integer between 0 and $i-1$. We pick $j:=\max \left\{\left\lfloor s_{i} / 8\right\rfloor, 1\right\}$. Since $i \geq s_{i}$, we get for $i \in J_{t}$,

$$
p_{i} \geq 1+\frac{\left(\frac{i-1}{2}\right)^{j}}{j^{j}}\left(\frac{s_{i}}{i-1}\right)^{j} \geq 1+\left(\frac{s_{i}}{2 j}\right)^{j} \geq 2^{s_{i} / 8} \geq 2^{2^{t-4}} .
$$

Thus, we can write

$$
(16)=\sum_{i=1}^{\left|A_{v}\right|} \varepsilon_{i} \frac{\log p_{i}}{p_{i}}=\sum_{t=0}^{\log n} \sum_{i \in J_{t}} \varepsilon_{i} \frac{\log p_{i}}{p_{i}}=\sum_{t=0}^{\log n} \sum_{i \in J_{t}} O\left(\frac{\varepsilon_{i} 2^{t}}{2^{2^{t-4}}}\right) \leq \sum_{t=0}^{\log n} O\left(\frac{2^{2 t+1}}{2^{2^{t-4}}}\right)=O(1) .
$$


To bound (16), define $J_{t}$ as before and let $p_{i}=\prod_{j=1}^{i-1} 1 /\left(1-\varepsilon_{j}\right)$. Observe that the function $\log (1 /(1-x))$ is a convex function which means if $s_{i}:=\sum_{j=1}^{i-1} \varepsilon_{j}$ is fixed, then $\prod_{j=1}^{i-1} 1 /\left(1-\varepsilon_{j}\right)$ is minimized when $\varepsilon_{j}=s_{i} /(i-1)$. Thus,

$$
p_{i} \geq\left(\frac{1}{1-\frac{s_{i}}{i-1}}\right)^{i-1} \geq\left(1+\frac{s_{i}}{i-1}\right)^{i-1} \geq 1+\left(\begin{array}{c}
i-1 \\
j
\end{array}\right)\left(\frac{s_{i}}{i-1}\right)^{j}
$$

in which $j$ can be chosen to be any integer between 0 and $i-1$. We pick $j:=\max \left\{\left\lfloor s_{i} / 8\right\rfloor, 1\right\}$. Since $i \geq s_{i}$, we get for $i \in J_{t}$,

$$
p_{i} \geq 1+\frac{\left(\frac{i-1}{2}\right)^{j}}{j^{j}}\left(\frac{s_{i}}{i-1}\right)^{j} \geq 1+\left(\frac{s_{i}}{2 j}\right)^{j} \geq 2^{s_{i} / 8} \geq 2^{2^{t-4}} .
$$

Thus, we can write

$$
(16)=\sum_{i=1}^{\left|A_{v}\right|} \varepsilon_{i} \frac{\log p_{i}}{p_{i}}=\sum_{t=0}^{\log n} \sum_{i \in J_{t}} \varepsilon_{i} \frac{\log p_{i}}{p_{i}}=\sum_{t=0}^{\log n} \sum_{i \in J_{t}} O\left(\frac{\varepsilon_{i} 2^{t}}{2^{2^{t-4}}}\right) \leq \sum_{t=0}^{\log n} O\left(\frac{2^{2 t+1}}{2^{2^{t-4}}}\right)=O(1) .
$$

Analyzing (13) The analysis of this equation is very similar to (12). We can write (13) using (11) and (10). We also use the same technique as in analyzing (16).

$$
\begin{aligned}
& (13)=\sum_{i=1}^{\left|A_{v}\right|} \varepsilon_{i}^{\prime}\left(1-\varepsilon_{i}\right) \prod_{j=1}^{i-1}\left(1-\varepsilon_{j}\right)\left(1-\varepsilon_{j}^{\prime}\right)\left(\sum_{j=1}^{i} \log \frac{\log \frac{2 n}{\left|V_{a_{j}}^{w_{a_{i}}}\right|}}{\log \frac{2 n}{\left|V_{a_{j}}^{v}\right|}}\right)= \\
& \sum_{i=1}^{\left|A_{v}\right|} \varepsilon_{i}^{\prime}\left(1-\varepsilon_{i}\right) \prod_{j=1}^{i-1}\left(1-\varepsilon_{j}\right)\left(1-\varepsilon_{j}^{\prime}\right)\left(\sum_{j=1}^{i} \log \frac{\log \frac{2 n}{\left|V_{a_{j}}^{v}\right|}+\log \frac{1}{1-\varepsilon_{j}}}{\log \frac{2 n}{\left|V_{a_{j}}^{v}\right|}}\right)= \\
& \sum_{i=1}^{\left|A_{v}\right|} \varepsilon_{i}^{\prime}\left(1-\varepsilon_{i}\right) \prod_{j=1}^{i-1}\left(1-\varepsilon_{j}\right)\left(1-\varepsilon_{j}^{\prime}\right)\left(\sum_{j=1}^{i} \log \left(1+\frac{\log \frac{1}{1-\varepsilon_{j}}}{\log \frac{2 n}{\left|V_{a_{j}}^{v}\right|}}\right)\right) \leq \\
& \sum_{i=1}^{\left|A_{v}\right|} \varepsilon_{i}^{\prime}\left(1-\varepsilon_{i}\right) \prod_{j=1}^{i-1}\left(1-\varepsilon_{j}\right)\left(1-\varepsilon_{j}^{\prime}\right)\left(\sum_{j=1}^{i} \log \frac{1}{1-\varepsilon_{i}}\right) .
\end{aligned}
$$

Let $s_{i}:=\sum_{j=1}^{i} \varepsilon_{j}$, and $s_{i}^{\prime}:=\sum_{j=1}^{i-1} \varepsilon_{j}^{\prime}$. Similar to the previous case, let $J_{t}, t \geq 0$, be the set of indices such that for each $i \in J_{t}$ we have $2^{t}-1 \leq s_{i}+s_{i}^{\prime} \leq 2^{t+1}$. Also define $p_{i}=\prod_{j=1}^{i} 1 /\left(1-\varepsilon_{j}\right)$ and $p_{i}^{\prime}=\prod_{j=1}^{i-1} 1 /\left(1-\varepsilon_{j}^{\prime}\right)$. Using the previous techniques we can get that $p_{i} \geq 2^{s_{i} / 8}$ and $p_{i}^{\prime} \geq 2^{s_{i}^{\prime} / 8}$. We get

$(13) \leq \sum_{t=0}^{\log n} \sum_{i \in J_{t}} \varepsilon_{i}^{\prime} \frac{\log p_{i}}{p_{i} p_{i}^{\prime}} \leq \sum_{t=0}^{\log n} \sum_{i \in J_{t}} \varepsilon_{i}^{\prime} \frac{s_{i}}{8 \cdot 2^{s_{i} / 8} 2^{s_{i}^{\prime} / 8}} \leq \sum_{t=0}^{\log n} \sum_{i \in J_{t}} \varepsilon_{i}^{\prime} \frac{2^{t+1}}{8 \cdot 2^{\left(2^{t}-1\right) / 8}} \leq \sum_{t=0}^{\log n} \frac{2^{2 t+2}}{8 \cdot 2^{\left(2^{t}-1\right) / 8}}=O(1)$. 
Analyzing (14) Let $b \in\{0,1\}$ be such that $b \equiv \max _{v}$ and let $k:=\left|V_{\max _{v}+1}^{v}(b)\right|$. We use Lemma 19. Observe that we still have $\mathrm{Con}_{v}=\mathrm{Con}_{w_{\max }}$. Thus we have,

$$
\begin{aligned}
& (14) \leq \frac{\left(k-\mathrm{Con}_{v}\right)}{\left|V_{\max _{v}+1}^{v}\right|-\mathrm{Con}_{v}} \prod_{i=1}^{\left|A_{v}\right|}\left(1-\varepsilon_{i}\right)\left(1-\varepsilon_{i}^{\prime}\right)\left(\varphi\left(w_{\max _{v}}\right)-\varphi(v)\right)=
\end{aligned}
$$

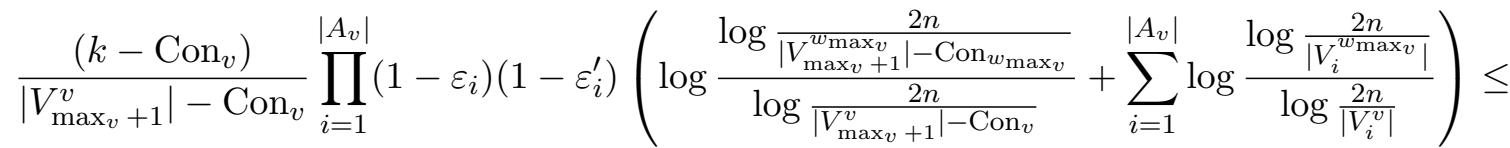

$$
\begin{aligned}
& \frac{\left(k-\operatorname{Con}_{v}\right)}{\left|V_{\max _{v}+1}^{v}\right|-\operatorname{Con}_{v}} \prod_{i=1}^{\left|A_{v}\right|}\left(1-\varepsilon_{i}\right)\left(1-\varepsilon_{i}^{\prime}\right)\left(\log \frac{\log \frac{2 n}{k-\operatorname{Con}_{v}}}{\log \frac{2 n}{\left|V_{\max _{v}+1}^{v}\right|-\operatorname{Con}_{v}}}+\sum_{i=1}^{\left|A_{v}\right|} \log \left(1+\log \frac{1}{1-\varepsilon_{i}}\right)\right) \leq \\
& \frac{\left(k-\mathrm{Con}_{v}\right)}{\left|V_{\max _{v}+1}^{v}\right|-\mathrm{Con}_{v}} \log \frac{\log \frac{2 n}{k-\mathrm{Con}_{v}}}{\log \frac{2 n}{\left|V_{\max _{v}+1}^{v}\right|-\mathrm{Con}_{v}}}+O(1)=O(1) \text {. }
\end{aligned}
$$

\section{C.2.3 Bounding (8)}

The big difference here is that the candidate set $V_{\max _{v}+1}^{w_{a}}$ becomes an active candidate set (if of course $\left.\max _{v}+1<n / 3\right)$ at $w_{a}$ while $V_{\max _{v}+1}^{v}$ was not active at $v$. Because of this, we have

$\varphi\left(w_{a}\right)-\varphi(v) \leq \log \log \frac{2 n}{\left|V_{a+1}^{w_{a}}\right|-\operatorname{Con}_{w_{a}}}+\log \log \frac{2 n}{\left|V_{\max _{v}+1}^{w_{a}}\right|}-\log \log \frac{2 n}{\left|V_{\max _{v}+1}^{v}\right|-\operatorname{Con}_{v}}+\sum_{j \in A_{v}} \log \frac{\log \frac{2 n}{\left|V_{j}^{w_{a}}\right|}}{\log \frac{2 n}{\left|V_{j}^{v}\right|}}$.

Thus, we get that

$$
\begin{aligned}
\sum_{a>\max _{v}}^{n / 3} \operatorname{Pr}\left[F\left(x_{v}\right)=\right. & \left.a \mid \Pi \in S_{v}\right]\left(\varphi\left(w_{a}\right)-\varphi(v)\right)= \\
& \sum_{a>\max _{v}}^{n / 3} \operatorname{Pr}\left[F\left(x_{v}\right)=a \mid \Pi \in S_{v}\right] \quad \log \log \frac{2 n}{\mid V_{a+1}^{w_{a} \mid-\operatorname{Con}_{w_{a}}}+} \\
& \sum_{a>\max _{v}}^{n / 3} \operatorname{Pr}\left[F\left(x_{v}\right)=a \mid \Pi \in S_{v}\right] \quad \log \left(\frac{\log \frac{2 n}{\left|V_{\max _{v}+1}^{w_{a}}\right|}}{\log \frac{2 n}{\left|V_{\max _{v}+1}^{v}\right|-\operatorname{Con}_{v}}}\right)+ \\
& \sum_{a>\max _{v}}^{n / 3} \operatorname{Pr}\left[F\left(x_{v}\right)=a \mid \Pi \in S_{v}\right] \quad \sum_{j \in A_{v}} \log \frac{\log \frac{2 n}{\left|V_{j}^{w a}\right|}}{\log \frac{2 n}{\left|V_{j}^{v}\right|}} .
\end{aligned}
$$


Using the previous ideas, it is easy to see that we can bound (19) as

$$
\begin{array}{r}
(19) \leq \sum_{a>\max _{v}}^{n / 3} \operatorname{Pr}\left[F\left(x_{v}\right)=a \mid \Pi \in S_{v} \wedge F\left(x_{v}\right)>\max _{v}\right] \cdot \operatorname{Pr}\left[F\left(x_{v}\right)>\max _{v} \mid \Pi \in S_{v}\right] \sum_{j \in A_{v}} \log \frac{\log \frac{2 n}{\left|V_{j}^{w_{a}}\right|}}{\log \frac{2 n}{\left|V_{j}^{v}\right|}} \leq \\
\sum_{a>\max _{v}}^{n / 3} \operatorname{Pr}\left[F\left(x_{v}\right)=a \mid \Pi \in S_{v} \wedge F\left(x_{v}\right)>\max _{v}\right] \cdot \prod_{i=1}^{\left|A_{v}\right|}\left(1-\varepsilon_{i}\right)\left(1-\varepsilon_{i}^{\prime}\right) \sum_{j \in A_{v}} \log \frac{\log \frac{2 n}{\left|V_{j}^{w_{a}}\right|}}{\log \frac{2 n}{\left|V_{j}^{v}\right|} \leq} \\
\prod_{i=1}^{\left|A_{v}\right|}\left(1-\varepsilon_{i}\right)\left(1-\varepsilon_{i}^{\prime}\right) \sum_{j \in A_{v}} \log \frac{\log \frac{2 n}{\left|V_{j}^{w_{a}}\right|}}{\log \frac{2 n}{\left|V_{j}^{v}\right|} \leq} \\
\prod_{i=1}^{\left|A_{v}\right|}\left(1-\varepsilon_{i}\right)\left(1-\varepsilon_{i}^{\prime}\right) \sum_{j \in A_{v}} \log \left(1+\log \frac{1}{1-\varepsilon_{j}}\right) \leq \\
\prod_{i=1}^{\left|A_{v}\right|}\left(1-\varepsilon_{i}\right) \sum_{j \in A_{v}} \log \frac{1}{1-\varepsilon_{j}} \leq \\
\prod_{i=1}^{\left|A_{v}\right|}\left(1-\varepsilon_{i}\right) \log \left(\frac{1}{\prod_{j \in A_{v}}\left(1-\varepsilon_{j}\right)}\right)
\end{array}
$$

To analyze (18) by Lemma 20 we know that $\operatorname{Pr}\left[F\left(x_{v}\right)>\max _{v} \mid \Pi \in S_{v}\right] \leq \frac{\left|V_{\max _{v}+1}^{v}\right|-k}{\left|V_{\max _{v}+1}\right|-\operatorname{Con}_{v}}$ in which $k$ is as defined in the lemma. Note that in this case $\left|V_{\max _{v}+1}^{w_{a}}\right|=\left|V_{\max _{v}+1}^{v}\right|-k$. This implies

$$
\begin{array}{r}
(18) \leq \sum_{a>\max _{v}}^{n} \operatorname{Pr}\left[F\left(x_{v}\right)=a \mid \Pi \in S_{v}\right] \log \left(\frac{\log \frac{2 n}{\left|V_{\max _{v}+1}^{v}\right|-k}}{\log \frac{2 n}{\left|V_{\max _{v}+1}^{v}\right|-\mathrm{Con}_{v}}}\right)= \\
\left(\sum_{a>\max _{v}}^{n} \operatorname{Pr}\left[F\left(x_{v}\right)=a \mid \Pi \in S_{v}\right]\right) \log \left(\frac{\log \frac{2 n}{\left|V_{\max _{v}+1}^{v}\right|-k}}{\log \frac{2 n}{\left|V_{\max _{v}+1}^{v}\right|-\mathrm{Con}_{v}}}\right)= \\
\operatorname{Pr}\left[F\left(x_{v}\right)>\max _{v} \mid \Pi \in S_{v}\right] \log \left(\frac{\log \frac{2 n}{\left|V_{\max _{v}+1}^{v}\right|-k}}{\log \frac{2 n}{\left|V_{\max _{v}+1}^{v}\right|-\mathrm{Con}_{v}}}\right) \leq \\
\frac{\left|V_{\max _{v}+1}^{v}\right|-k}{\left|V_{\max _{v}+1}^{v}\right|-\operatorname{Con}_{v}} \log \left(\frac{\log \frac{2 n}{\left|V_{\max _{v}+1}^{v}\right|-k}}{\log \frac{2 n}{\left|V_{\max _{v}+1}^{v}\right|-\mathrm{Con}_{v}}}\right)=O(1) .
\end{array}
$$

It is left to analyze (17). Let $x_{0}=\left|P_{0}^{v}\right|$ and $x_{1}=\left|P_{1}^{v}\right|$. Let $b_{i}$ be a Boolean such $b_{i} \equiv i$. Note that we have $\left|V_{\max _{v}+a+1}^{w_{\max _{v}+a} \mid}\right|=x_{\max _{v}+a}=n-x_{b_{\max }+a+1}$. Using Corollary 22 we can write 
(17) as follows:

$$
\begin{aligned}
(17) & \leq \sum_{a=1}^{n / 3-\max _{v}} \operatorname{Pr}\left[F\left(x_{v}\right)=\max _{v}+a \mid \Pi \in S_{v}\right] \log \log \frac{2 n}{\left|V_{\max _{v}+a+1}^{w_{\max }+a}\right|-\operatorname{Con}_{w_{\max _{v}+a}}} \\
& \leq \sum_{a=1}^{n / 3-\max _{v}} 2^{-\Omega(a)}\left(1-\frac{x_{b_{\max _{v}+a+1}}-\left\lfloor\left(\max _{v}+a+1\right) / 2\right\rfloor}{n-\max _{v}-a}\right) \log \log \frac{2 n}{n-x_{b_{\max _{v}+a+1}}-\left\lfloor\left(\max _{v}+a\right) / 2\right\rfloor} \\
& =O(1) .
\end{aligned}
$$

